UNIVERSIDAdE DE SÃo PAULO

Faculdade de EConomia, Administração e Contabilidade de Ribeirão Preto DePartamento de ECONOMia

Programa de Pós-GraduaÇão em Economia - Área: Economia APlicada

ADRIANO BARASAL MORALES

Localização industrial: uma aproximação usando processos pontuais espaciais

Orientador: Prof. Dr. Márcio Poletti Laurini

Ribeirão Preto

2018 
Prof. Dr. Vahan Agopyan

Reitor da Universidade de São Paulo

Prof. Dr. Dante Pinheiro Martinelli

Diretor da Faculdade de Economia, Administração e Contabilidade de Ribeirão Preto

Prof. Dr. Renato Leite Marcondes

Chefe do Departamento de Economia

Prof. Dr. Sergio Naruhiko Sakurai

Coordenador do Programa de Pós-Graduação em Economia 
ADRIANO BARASAL MORALES

\section{Localização industrial: uma aproximação usando processos pontuais espaciais}

Dissertação de mestrado apresentada ao Programa de Pós-Graduação em Economia - Área: Economia Aplicada da Faculdade de Economia, Administração e Contabilidade de Ribeirão Preto da Universidade de São Paulo, para obtenção do título de Mestre em Ciências. Versão Corrigida. A original encontra-se disponível no Serviço de Pós-Graduação da FEA-RP/USP.

Orientador: Prof. Dr. Márcio Poletti Laurini

Ribeirão Preto 
Autorizo a reprodução e divulgação total ou parcial deste trabalho, por qualquer meio convencional ou eletrônico, para fins de estudo e pesquisa, desde que citada a fonte.

MORALES, Adriano Barasal.

Localização industrial: uma aproximação usando processos pontuais espaciais / Adriano Barasal Morales - Ribeirão Preto, SP, 2018.

54 p.: il.; $30 \mathrm{~cm}$

Dissertação de mestrado apresentada ao Programa de PósGraduação em Economia - Área: Economia Aplicada da Faculdade de Economia, Administração e Contabilidade de Ribeirão Preto da Universidade de São Paulo, para obtenção do título de Mestre em Ciências. - Universidade de São Paulo

Orientador: Laurini, Márcio Poletti

1. Localização Industrial. 2. São Paulo. 3. Estatística Espacial. 


\title{
Localização industrial: uma aproximação usando processos pontuais espaciais
}

\begin{abstract}
Projeto de dissertação para a obtenção do título de mestre em ciências econômicas do Departamento de ciências econômicas de Ribeirão Preto da Universidade de São Paulo
\end{abstract}

Trabalho aprovado. Ribeirão Preto, São Paulo, 2017

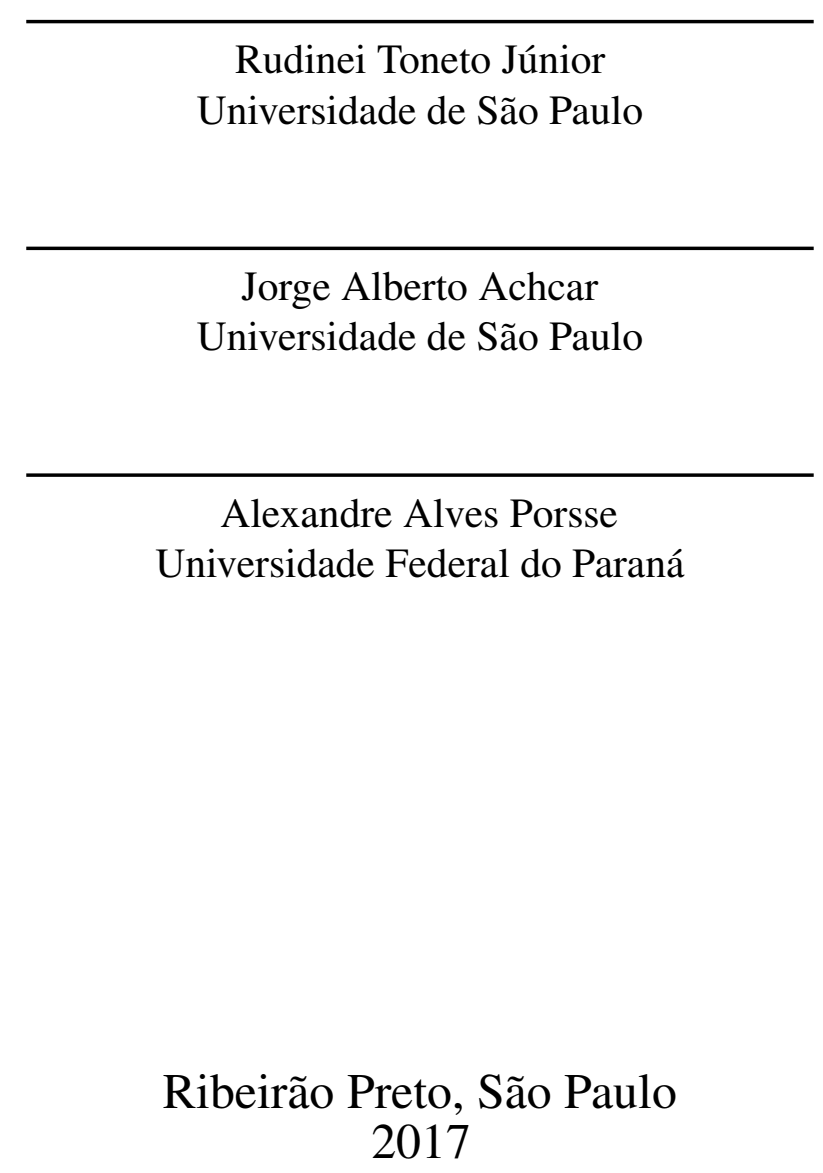





\section{Resumo}

MORALES, A. B. Localização industrial: uma aproximação usando processos pontuais espaciais. Dissertação (Mestrado) - Faculdade de Economia, Administração e Contabilidade de Ribeirão Preto, Universidade de São Paulo, Ribeirão Preto, 2017.

O objetivo desta pesquisa é mostrar como aproveitar novas bases de dados disponíveis e o avanço de métodos computacionais para extrair informações estatísticas sobre a localização espacial de firmas. Para isso, propomos uma aplicação de métodos de estatística espacial para modelar o padrão de localização de novas empresas de serviços no município de São Paulo. Neste trabalho, assumimos que a localização espacial dessas firmas foi gerada através de um processo pontual bidimensional e assim aplicamos dois modelos distintos: um baseado em intensidade não estocástica baseada no processo de Poisson, e um modelo de intensidade estocástica baseado processo de Cox log Gaussiano (Log Gaussian Cox Process - LGCP). A principal base de dados utilizada é base georeferenciada baseada no Cadastro Central de Empresas construída pelo Centro de Estudos da Metrópole (CEM), contendo observações de empresas na região metropolitana de São Paulo, para o ano base de 2000. Utilizamos como variáveis explicativas de localização informações advindas de sistemas de informações geográficas (SIG), o Censo demográfico e imagens de satélite do National Oceanic and Atmospheric Administration (NOAA). Os resultados encontrados mostram a importância dessa metodologia no processo de construção de modelos de localização espacial, combinando distintas fontes de dados e introduzindo novas perspectivas sobre o estudo empírico de economia urbana.

Palavras-chave: Localização de firmas, São Paulo, estatística espacial, processo pontual de Poisson, LGCP, INLA. 



\section{Abstract}

MORALES, A. B. Firm location: an approach using spatial point process. Dissertation (Master Degree) - School of Economics, Business and Accounting at Ribeirão Preto, University of São Paulo, Ribeirão Preto, 2017.

The objective of this research is to show how to take advantage of new available databases and computational methods to extract statistical information about the spatial location of firms. In this sense, we propose an application of spatial statistics methods to model the location patterns of new services firms in the city of São Paulo. In this paper, we assume that the spatial location of these firms was generated through a two-dimensional point process and thus we applied two distinct models: one based on non-stochastic intensity based on the Poisson process, and a stochastic intensity model based on the Log Gaussian Cox process (LGCP). The main input used is a georeferenced database based on the Central Business Register made by the Center for Metropolis Studies (CEM), containing data of firms in the metropolitan region of São Paulo, for the base year 2000. We use as explanatory variables information from geographic information systems (GIS), demographic census and satellite imagery from National Oceanic and Atmospheric Administration (NOAA). The results show the usefulness of these models the construction of spatial location models, combining different data sources and introducing new perspectives on the empirical study of urban economics.

Keywords: location of firms, São Paulo, spatial statistics, Poisson point process, LGCP, INLA. 



\section{Lista de ilustrações}

Figura 3.1 - Realização do processo pontual para empresas de serviços. . . . . . . . . . 24

Figura 3.2 - Imagem de iluminação noturna da região de interesse. . . . . . . . . . . . . 25

Figura 3.3 - Intensidade de linhas de ônibus calculada usando Kernel ajustado para as

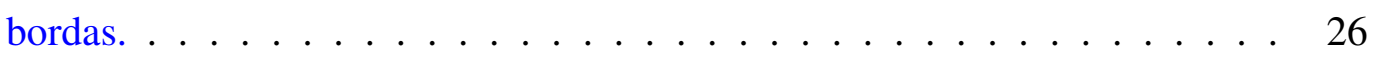

Figura 3.4 - Intensidade de empresas até o ano de $1999 \ldots$. . . . . . . . . . . . . 28

Figura 5.1 - Estimativa da intensidade não-paramétrica da localização das novas empresas no ano 2000, usando Kernel ajustado para efeitos de borda. . . . . . . . . . 37

Figura 5.2 - Contagem de firmas no grid utilizado na estimação. . . . . . . . . . . . . 38

Figura 5.3 - Ajuste do modelo de Poisson irrestrito no município de São Paulo. . . . . . 40

Figura 5.4 - Erro do modelo de Poisson irrestrito no município de São Paulo. . . . . . . 41

Figura 5.5 - Ajuste - LGCP irrestrito. . . . . . . . . . . . . . . . . . . . 43

Figura 5.6 - Efeito estruturado capturado pelo modelo LGCP irrestrito. . . . . . . . . . . 43

Figura 5.7 - Efeito não estruturado capturado pelo modelo LGCP irrestrito. . . . . . . . . 44

Figura 5.8 - Erro do modelo LGCP irrestrito: predito menos observado. . . . . . . . . . 44

Figura 5.9 - Ajuste - LGCP irrestrito. . . . . . . . . . . . . . . . . . . 46

Figura 5.10-Efeito estruturado capturado pelo modelo LGCP restrito. . . . . . . . . . . 46

Figura 5.11-Efeito não estruturado capturado pelo modelo LGCP restrito. . . . . . . . . . 47

Figura 5.12-Erro do modelo LGCP restrito: predito menos observado. . . . . . . . . . . 47 


\section{Lista de tabelas}

Tabela 5.1 - Estatística Descritiva - Variáveis no Grid Estimado . . . . . . . . . . . . . 38

Tabela 5.2 - Estimação dos parâmetros do modelo de Poisson . . . . . . . . . . . . . . . 39

Tabela 5.3 - Estimação dos parâmetros do modelo LGCP irrestrito . . . . . . . . . . . . 41

Tabela 5.4 - Estimação dos hiperparâmetros dos efeitos aleatórios do modelo LGCP irrestrito 41

Tabela 5.5 - Estimação dos parâmetros do modelo LGCP restrito . . . . . . . . . . . . . 45

Tabela 5.6 - Estimação dos hiperparâmetros dos efeitos aleatórios do modelo LGCP restrito 45

Tabela 5.7 - Estatística de ajuste dentro da amostra . . . . . . . . . . . . . . 48 


\section{Sumário}

$1 \quad$ INTRODUÇÃo $\ldots \ldots \ldots \ldots \ldots \ldots \ldots \ldots$

2 ABORDAGEM ESPACIAL EM ECONOMIA URBANA $\ldots \ldots \ldots$

$3 \quad$ DESCRIÇÃO DOS DADOS $\ldots \ldots \ldots \ldots \ldots \ldots$

4 METODOLOGIA . . . . . . . . . . . . . 29

4.1 Processos pontuais e o modelo de Poisson . . . . . . . . 29

$4.2 \quad$ Modelo LGCP e a estimação por INLA . . . . . . . . . . . . . . 32

$5 \quad$ APLICAÇÃO EMPÍRICA $\ldots \ldots \ldots \ldots \ldots \ldots \ldots \ldots$

$6 \quad$ CONCLUSÃo $\ldots \ldots \ldots \ldots \ldots \ldots \ldots \ldots \ldots \ldots \ldots$

REFERÊNCIAS . . . . . . . . . . . 51 



\section{Introdução}

Novos métodos de construção de bases de dados e modelagem espacial tem possibilitado a construção de dados de alta qualidade acerca do espaço geográfico, e também análises estatísticas com alta complexidade e realismo. Os sistemas de análise espacial são mais conhecidos como Sistemas de Informações Geográficas (SIG), e o uso dessas ferramentas já é recorrente e bastante avançada em diversas literaturas como em meteorologia (Chapman e Thornes (2003)) e na área da saúde (Carvalho e Souza-Santos (2005)). No entanto, na literatura econômica, seu uso é mais recente e ainda bastante limitado a apenas alguns tipos de modelagens espaciais, normalmente associadas aos chamados modelos de econometria espacial, baseados em modelos areais discretos: ver por exemplo Goodchild, Anselin e Deichmann (1993) e Messner e Anselin (2004).

No contexto de economia urbana, pouco ainda é visto das técnicas mais avançadas que possam trabalhar com essas ferramentas e com essa disponibilidade de dados. Os trabalhos de modelagem econométrica espacial são normalmente baseados em modelos de regressão modificados para dados referentes a regiões discretas do espaço, como estados e municípios. Geralmente, estes trabalhos utilizam matrizes de ponderação espacial e estruturas de vizinhança discreta em modelos de autoregressões espaciais ou em modelos de erro espacial e formas derivadas destas estruturas. No entanto, estes modelos discretos não podem ser diretamente utilizados para modelar a ocorrência de eventos no espaço, no que é conhecido como processos pontuais. Processos pontuais são estruturas probabilísticas que determinam a intensidade da ocorrência de eventos no espaço de interesse, o que no caso de processos econômicos podem ser utilizados para representar os determinantes econômicos da presença de empresas no espaço.

Historicamente, no Brasil, os primeiros estudos que analisavam o perfil de empresas nos estados ou em regiões metropolitanas foram realizados por historiadores econômicos que se limitavam a agregar dados e fazer análises descritivas das bases de dados disponíveis. Exemplos desse método podem ser vistos em ARAÚJO (1999) e em RIBEIRO e ALMEIDA (1980). Outros trabalhos com técnicas um pouco mais avançadas já contavam com análises econométricas para estudar a localização de empresas em estados brasileiros (Hansen (1987), Resende (2015)). Mesmo em estudos feito fora do Brasil, a análise espacial ainda é limitada com relação aos métodos utilizados (Arauzo-Carod, Liviano-Solis e Manjón-Antolín (2010)), pois boa parte deles se baseiam apenas em análises de econometria espacial tradicional (Glaeser (2008)).

Essas técnicas, apesar de importante fonte de informações, ainda são incapazes de explorar toda a riqueza dos dados econômicos, principalmente por lidarem apenas com dados agregados, o que gera perda de informação para fazer inferência estatística (Orcutt, Watts e Edwards (1968)). No entanto, mais recentemente alguns estudos econômicos passaram a analisar a localização industrial por meio de técnicas estatísticas com dados espaciais desagregados aproveitando também as novas informações disponíveis pelos SIG (Bocci e Rocco (2016)), o que enriqueceu 
bastante a literatura empírica.

Partindo desse contexto mais recente, pretendemos continuar expandindo o uso dessas técnicas estatísticas no contexto de localização industrial. Quer-se mostrar como a riqueza de informações espaciais disponível podem ser utilizadas para realizar a modelagem do processo pontual de localização espacial de empresas. Nesta pesquisa, propomos uma aplicação a esse tipo de dados em economia urbana. Ou seja, pretende-se combinar imagens espaciais do tipo "raster", contendo informações projetadas continuamente no espaço, com dados censitários em Tabela e dados espaciais no formato de "shapefile", e analisar essa base de dados através de modelos paramétricos de processos pontuais da classe Poisson e Cox Log Gaussiano (LGCP). Para tanto, assumiremos que a localização espacial de cada firma de serviço orientado para negócios no município de São Paulo é resultado de um mecanismo estocástico. Isto é, são observações geradas por um processo pontual no sentido descrito por Baddeley, Bárány e Schneider (2007).

Como discutido acima, propomos neste trabalho dois modelos diferentes. No primeiro deles, vamos modelar os dados espaciais de empresas de serviços em São Paulo usando um modelo de processos pontuais de Poisson (Baddeley, Rubak e Turner (2015)). Este é um modelo aditivo onde a intensidade de ocorrência é determinística, i.e., as covariadas entram de forma determinística na equação da intensidade do processo, aumentando ou diminuindo-a. O processo de Poisson é o modelo padrão de modelagem de ocorrência de processos pontuais, e tem várias vantagens em relação as abordagens usuais que utilizam modelos para dados discretos.

Primeiro, não é preciso mais agregar os dados usando construções artificiais como limites censitários para construir o modelo, e assim cada observação será analisada no continuum espacial. Segundo, propiciaremos novas informações econômicas até então indisponíveis com esse conjunto de dados, já que estas informações também são baseadas em mensurações contínuas no espaço, possibilitando explorar e misturar bases de dados disponíveis há muito tempo e que ainda não foram trabalhadas dessa forma. Dessa forma, os métodos que estamos utilizando se destoam do que vinha sendo feito na literatura empírica deste tema.

No entanto, o modelo de Poisson apresenta algumas possíveis limitações. Em especial, o modelo de Poisson apresenta uma propriedade conhecida como independência condicional, i.e., condicionalmente as variáveis explicativas do modelo, as realizações do processo são independentes. Essa limitação é importante já que o modelo Poisson só permite capturar efeitos de aglomeração e clustering via fatores condicionantes, e dessa forma mecanismos de atração não capturados por variáveis explicativas não são adequadamente modelados.

Já o segundo modelo, conhecido como processo de Cox Log Gaussiano (Log Gaussian Cox Process - LGCP), é uma extensão do primeiro e do processo de Cox, pois captura os efeitos não observados que não estão no modelo de efeitos determinísticos (Møller, Syversveen e Waagepetersen (1998)). Dessa forma, o modelo incorpora, além do conjunto de variáveis explicativas que capturam os efeitos fixos, a presença de efeitos aleatórios também: ver por exemplo Diggle (1983), Cressie (1993) e Chiu et al. (2013).

A vantagem em utilizar esta variação do processo de Cox é que o modelo LGCP consegue 
capturar estruturas espaciais em diferentes escalas dentro do mesmo modelo (Illian, Sørbye e Rue (2012)). Em especial, capturar efeitos especiais não observados é uma aproximação utilizada para detectar efeitos de atração ou repulsão que são independentes dos efeitos fixos. Em economia urbana, apesar de ser muito conhecido o chamado efeito de clustering industrial (Gordon e McCann (2000)), ele é muito difícil de ser modelado empiricamente. Assim, utilizar os efeitos espaciais do modelo LGCP pode ser uma ótima aproximação para capturar este efeito.

Outro avanço metodológico que esta pesquisa faz é a estimação Bayesiana de ambos os modelos utilizando a metodologia de aproximações integradas aninhadas de Laplace (Integrated Nested Laplace Approximations - INLA) (Rue, Martino e Chopin (2009)1) ${ }^{1.1}$. Este método já é bastante utilizado em áreas do conhecimento como meteorologia e biologia, mas é particularmente novo na área de economia urbana. A ferramenta INLA fornece um método conveniente de obter aproximações de Laplace para as marginais posteriores dos parâmetros dos modelos bayesianos hierárquicos. Isso é possível sempre que os efeitos latentes forem expressos como Campos aleatório Gaussiano Markoviano (Gaussian Markov Random Field - GMRF). As aproximações de Laplace, quando comparadas com métodos tradicionais de aproximações numéricas como Monte Carlo via Cadeia de Markov (MCMC), geram imenso ganho computacional, já que são baseadas em aproximações determinísticas com elevada acurácia, evitando os problemas de convergência e o tempo computacional envolvido em métodos baseados em simulação para processos de inferência Bayesiana. Isso permite que modelos com estruturas complexas possam ser estimados. Dessa forma, a introdução desses modelos estimados com INLA na literatura de economia urbana é um importante avanço. Pois, além de poder produzir importantes resultados, abre a possibilidade para futuras extensões metodológicas nesta literatura.

Sendo assim, implementamos nesta pesquisa dois modelos importantes e complexos para a análise de dados. Embora tenhamos informações novas com a implementação do modelo em uma região importante como o município de São Paulo, o mais importante é a expansão dos métodos empíricos que está sendo proporcionada com esta pesquisa. Assim, lançar novas informações e perspectivas sobre o tema é o principal objetivo deste trabalho empírico.

O que motiva a explorar novos métodos e dados espaciais com a intenção de encontrar padrões espaciais para a indústria é a relação deste tema com a gestão pública. Políticos e gestores públicos estão diretamente interessados em entender o que faz empresas de um determinado segmento se instalarem em uma região e não e outra. Essa importante necessidade é o que movimenta este campo empírico na área de economia urbana. Prover esse tipo de informação auxilia os gestores públicos em suas tarefas de atrair investimento, renda e empregos para certas áreas. Isto é, possibilita o desenvolvimento de certas regiões urbanas ao mudar os incentivos espaciais de localização das empresas. Isso acontece dado que as empresas respondem a incentivos espaciais, i.e, por conta do comportamento maximizador de lucro as empresas escolhem sua localização de forma a existir um equilíbrio espacial (Brueckner (1987)). Sendo

$\overline{1.1}$ O modelo de Poisson é geralmente estimado pelo método de máxima pseudoverossimilhança, mas optamos por estimá-lo por INLA neste trabalho. Na seção de metodologia acrescentamos uma breve explicação sobre o método de máxima pseudoverossimilhança. 
assim, pretendemos lançar algumas respostas e novas perspectivas ao tema através desse trabalho empírico. Na próxima seção explicaremos melhor sobre o comportamento de localização das empresas ao resumir algumas das principais teorias em economia urbana.

Dessa forma, concentramos esse estudo em empresas de serviços por estarmos trabalhando com dados localizados em uma cidade altamente urbanizada, São Paulo (Kolko (2010)) e também por ser um segmento de alto valor e importância agregados. Existem poucos trabalhos que analisam o perfil das indústrias ou de suas localizações espaciais neste município. A maioria deles aproveitam os dados da Paep ${ }^{1.2}$ para traçar características descritivas das indústrias do estado ou da região metropolitana (Fernandes, Côrtes e Pinho (2004)), usando dados agregados. Por outro lado, esta pesquisa pretende explorar outras bases de dados que permitem o uso da modelagem de processos pontuais, o que representa um avanço em técnicas espaciais.

Estruturamos o trabalho da seguinte maneira: A Seção 2 vai aproximar a discussão de Economia Urbana com este trabalho empírico, indicando o porquê de se utilizar esses modelos espaciais e a seleção de covariadas, a Seção 3 descreverá os dados que estamos utilizando para fazer a pesquisa, a Seção 4 apresentará a metodologia, a Seção 5 terá a aplicação empírica e os resultados e por fim na última seção faremos algumas considerações finais do trabalho.

$\overline{1.2}$ Pesquisa da Atividade Econômica Paulista - Disponível em: < http://produtos.seade.gov.br/produtos/paep/> Acesso em: 01/10/2017 


\section{Abordagem espacial em Economia Urbana}

Nesta seção pretende-se fazer um breve panorama histórico da modelagem espacial em economia urbana. Faremos isso para aproximar o que está sendo desenvolvido neste trabalho com a literatura teórica do tema. Ao fazer isso, fica fácil mostrar a necessidade de ferramentas cada vez mais complexas para extrair as informações neste campo de estudo, como essa que motiva a nossa pesquisa. Dessa forma, muitos modelos importantes desenvolvidos na literatura serão apresentados enquanto fazemos um paralelo entre este trabalho empírico e a teoria.

A evolução do tema passa pela tradição clássica e neoclássica, em que as firmas manufatureiras são agentes econômicos atomizados maximizadores de lucro, chegando até as literaturas mais recentes que estudam modelos de aglomerações (Duranton e Puga (2004)) e modelos de equilíbrio de cidade (Lucas e Rossi-Hansberg (2002)). A ênfase em manufatura está relacionada com a época em que esses modelos foram criados, início do séc. XX. Neste caso, os serviços não tinham tanta influência e grandes linhas de montagem se destacavam na manufatura. Além disso, acreditava-se que a indústria de serviços não gerava bens transacionáveis (Barlet, Briant e Crusson (2013)). Já os estudos que lidam com aglomerações, ou coaglomerações, relacionam boa parte da vida urbana, discutindo a localização não só da atividade econômica mas também das pessoas que habitam o espaço urbano ${ }^{2.1}$. Nesta seção, que conta com uma breve trajetória do tema, nos concentraremos somente em localização industrial. Já a localização das pessoas não faz parte do escopo deste trabalho.

A preocupação com a localização espacial das firmas é um tema antigo. Primeiramente abordado pelos economistas clássicos, o modelo formalizado por Weber (1909) é ponto de partida para muitos outros mais complexos. Neste modelo, as empresas estão preocupadas com os custos de transporte, e se localizam de maneira ótima no ponto em que minimizam o custo de transporte dos insumos e também de seu produto até o mercado consumidor. De maneira mais simples, o modelo considera o mercado como alguns pontos no espaço e não como um continuum. Mas mesmo com suas limitações, o modelo introduziu conceitos de maximização de lucros para o conceito espacial, servindo de base para os mais complexos.

Ainda neste arcabouço teórico, Moses (1958) conseguiu encaixar a teoria de localização industrial ótima nos problemas clássicos de microeconomia. $\mathrm{O}$ autor mostra que a empresa, ao maximizar o lucro restrita a um orçamento, está ao mesmo tempo decidindo aonde se localizar no espaço de maneira a minimizar os custos de transportes. Logo, localização espacial faz parte do problema da firma assim como atingir a isoquanta de produção mais alta dada a restrição orçamentária.

2.1 O artigo que estamos utilizando como referência por servir como base para a vida urbana e o efeito de economia de aglomeração é de Helsley e Strange (2014). 
Em um primeiro momento, essas teorias econômicas foram importantes para introduzir o comportamento espacial das firmas. Mesmo que elas lidem com a empresa de forma atomizada, sem se preocupar com aglomerações espaciais, já se torna possível justificar que existem padrões espaciais nos quais as firmas se baseiam para escolher sua localização ótima. Esse discernimento é crucial para boa parte das pesquisas empíricas que foram feitas em economia urbana. Isto é, o comportamento econômico está por trás da localização espacial das firmas.

Essa linha de pensamento, embora reveladora, não é capaz de esclarecer todos os fenômenos observados nos dados espaciais. É muito comum observar aglomerações espaciais de indústrias enquanto em outros locais é normal ver dispersões. Os dados usados neste estudo também mostram esse padrão, e o motivo da aglomeração e de dispersão é objeto de estudo no modelo que será apresentado adiante. A teoria em economia urbana também tratou de estudar esses padrões da atividade econômica.

A ideia de economia de aglomeração está relacionada a existência de externalidades positivas em se habitar cidades, onde há uma grande concentração de habitantes e setores produtivos. Caso contrário, pessoas se dispersariam em regiões de custos mais baixos que os encontrados em áreas urbanas. Ou seja, há uma ideia de retornos crescentes de produtividade em uma região de alta densidade econômica. Ciccone e Hall (1996), por exemplo, mostraram a relação entre densidade de atividade econômica e produtividade advinda por efeitos de externalidades em economias de aglomeração. Outra externalidade positiva constatada em economias de aglomeração foi encontrada no grau de ensino dos trabalhadores de diferentes cidades (Moretti (2004)).

Aglomerações já eram conhecidas pelos clássicos, mas quem formalizou este conceito foi Marshall (1890). Em sua literatura, o que caracterizava as aglomerações de atividade econômica são fatores externos à uma simples firma, mas que afetam todas as firmas em uma dada região. Para o autor, empresas de uma determinada indústria se aglomeram pois se beneficiam de fatores intangíveis tais como o efeito de transbordamento de conhecimento, insumos locais não-transacionáveis e pela fonte de trabalho qualificado gerado pela aglomeração de empresas. Ou seja, fatores que diminuiriam os custos de localização das firmas, compensando o fator congestão de empresas idênticas e o aumento dos preços dos insumos, causada pela alta demanda dada a grande quantidade de firmas na região.

No entanto, a limitação da teoria de Marshall está em pensar apenas em firmas de uma determinada indústria, enquanto que em uma área urbana vemos uma grande quantidade de diferentes indústrias aglomeradas. De forma a descrever melhor as economias de aglomeração, Hoover (1937), Hoover (1948) e Ohlin (1933) classificaram alguns tipos de economias de aglomeração que serviram de base para muitos outros trabalhos empíricos. Entre essas classificações, existem três tipos de causa para aglomerações de diversas atividades econômicas: retorno de escala interno, economia de localização e, por fim, economia de urbanização. Obviamente, depois desses tipos, muitos outros foram teorizados para tentar explicar outros fenômenos causadores de aglomeração da atividade econômica ${ }^{2.2}$.

\footnotetext{
$\overline{2.2}$ Demais modelos não fazem parte do escopo desse trabalho, mas podemos citar alguns para efeito de ilustração.
} 
Além do vasto escopo teórico produzido ao longo de séculos sobre economias de aglomerações, há fortes evidências empíricas que corroboram com esta literatura. Estudos empíricos recentes mostram que, além dos efeitos de aglomeração se dissiparem com a distância do centro urbano, há também efeitos naturais e diversas outras fontes comumente vistas em centros urbanos gerando esse efeito (Rosenthal e Strange (2004)). Já Greenstone, Hornbeck e Moretti (2010) viu evidência de efeitos de aglomerações em comparar mudanças na produtividade total dos fatores em municípios "vencedores", no sentido de atrair muitas indústrias, e municípios "fracassados"que atraíram poucas indústrias. No primeiro grupo houve grande acréscimo na produtividade total dos fatores de produção em comparação com o segundo grupo devido ao efeito de aglomeração de indústrias nas cidades. Além das forças que geram aglomerações, outros estudos mais modernos analisam empiricamente efeitos de aglomerações à luz das teorias de cidade: ver por exemplo Ellison, Glaeser e Kerr (2010), Behrens et al. (2017),Desmet e Rossi-Hansberg (2013) e Desmet e Rossi-Hansberg (2014)

O trabalho que está sendo realizado nesta pesquisa empírica leva em consideração esses principais aspectos teóricos e empíricos que foram apresentados. Só foi possível estudar os padrões de localização das firmas de serviço em São Paulo pois assumimos que as mesmas possuem tal comportamento espacial. Isto é, o modelo que usamos faz sentido neste contexto se considerarmos que as firmas estão preocupadas com a escolha de sua localização de forma racional, maximizando os lucros. Além disso, as covariadas que serão debatidas no modelo estão de acordo com as teorias de aglomerações urbanas. Ou seja, estamos querendo identificar os principais fatores de atração ou de repulsão das firmas na cidade. Na seção de metodologia, voltaremos a discorrer mais sobre as covariadas e a importância delas no modelo apresentado. No entanto, antes de descrevermos toda a estatística que será utilizada, a próxima seção descreverá os dados que compõe a pesquisa.

Entre eles, o modelo de pólo de crescimento, o modelo de incubação, o modelo de ciclo de produto, o modelo de Porter, o modelo de novas áreas indústriais entre outros. 



\section{Descrição dos dados}

Este trabalho conta com dados de diversos formatos e fontes diferentes, sendo que uni-los é um dos propósitos dessa pesquisa. Grande parte dos dados que compõe esta análise estão em formato shapefile, o que diferencia sua análise do padrão clássico de dados em Tabela (Bivand et al. (2008)). Esta categoria de dados, formada por linhas, traços, pontos ou polígonos pode ser melhor descrita através de plotagens do que Tabelas. Outros dados presentes são imagens do tipo rasters, que representam informações projetadas no contínuo espacial. Por fim, aproveitando toda essa estrutura espacial, agregaremos alguns dados censitários para que observações em formato de Tabela possam entrar como uma estrutura discreta na nossa janela de processo. A disposição de dados nesta estrutura espacial está intimamente ligada com o tipo de modelagem que será feita.

Os principais dados espaciais que serão utilizados foram extraídos da base de domínio público do Centro de Estudos da Metrópole (CEM). O trabalho da equipe do CEM consistiu basicamente em georreferenciar as empresas cadastradas no Cadastro Central de Empresas (CEMPRE), sendo 2000 o ano base da divulgação deste trabalho. Dessa forma, todos os dados que usaremos terão este mesmo ano base para que haja controle dos efeitos intertemporais.

Carregando a estrutura desses dados em formato shapefile, fizemos uma filtragem, por meio de códigos do CNAE, para trabalharmos apenas com empresas de serviços orientados para negócios. ${ }^{3.1}$ Dessa forma, de 452.816 registros na base, serão utilizadas apenas 1069 empresas que seguem essa classificação e que foram registradas no ano de 2000.

É possível ver como essas empresas estão dispostas na janela do processo na Figura 3.1.

A escolha por usar apenas o ano mais recente disponível na base de dados se deve ao fato de estarmos interessados nas empresas que foram criadas no ano base de 2000. Demais empresas desse mesmo segmento que surgiram nos anos anteriores também foram utilizadas, mas como covariadas do modelo conforme mostrado na seção anterior.

Outros shapefiles que serão incorporados a estrutura de dados também vieram do trabalho disponibilizado pelo CEM. Entre eles, vamos utilizar parte do mapa de logradouros do município de São Paulo e o mapa das linhas de ônibus para compor algumas das covariadas. Ambos constituídos por linhas e traços. Já o shapefile de polígonos que usamos para construir a janela do processo, o município de São Paulo, veio do site do IBGE ${ }^{3.2}$.

Além desses shapefiles, vamos sobrepor imagens do tipo raster para compor a base de dados desse trabalho. O principal arquivo desse tipo que entrará no modelo como covariada é a

3.1 A Classificação Nacional de Atividades Econômicas (CNAE) é um código utilizado pelo IBGE na qual toda firma legalizada possui para especificar sua principal atividade desenvolvida. As empresas que estamos utilizando neste trabalho seguem os códigos: 65.10-2, 65.21-8, 65.22-6, 65.23-4, 65.24-2, 66.11-7, 66.12-5, 66.13-3,66.21-4, 66.22-2, 67.11-3, 67.12-1, 67.19-9, 67.20-2, 70.10-6, 70.20-3, 71.10-2, 71.21-8, 71.22-6, 71.23-4, 72.10-9, 72.20-6, 73.10-5, 73.20-2, 74.11-0, 74.12-8, 74.13-6, 74.14-4, 74.15-2, 74.16-0, 74.20-9.

3.2 Disponível em: <https://mapas.ibge.gov.br/> Acesso em: 06/10/2017 


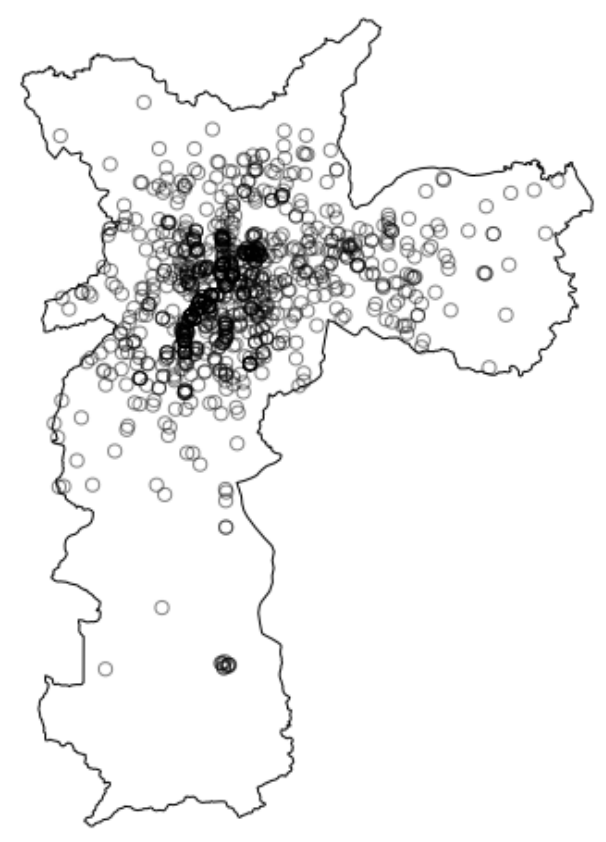

Figura 3.1 - Realização do processo pontual para empresas de serviços.

imagem noturna de satélite para o município de São Paulo no ano 2000, encontrada na página do NOAA $^{3.3}$. A importância desses dados será melhor apresentada a seguir quando definirmos as covariadas e como as incluiremos no modelo ${ }^{3.4}$.

Outros dados importantes que compõe a análise espacial se encontram em Tabelas. Certas informações encontradas em nível individual no Censo demográfico do IBGE fazem sentido no modelo de localização espacial, conforme especificado na seção anterior. Por isso trouxemos para análise os dados de renda individual de cada habitante de São Paulo no ano 2000 com o interesse de criar uma covariada para este tipo de dado. Porém, para incluir esse tipo de formato de dado na base de dado espacial, precisamos criar um certo nível de agregação. Por isso, dividimos em renda média por distrito censitário (a menor agregação disponível) de São Paulo para encaixar os dados como covariada do modelo espacial.

Já os dados que nos informam as linhas de metrô de São Paulo e da CPTM vieram do Google Maps em formato "tkml". Com isso concluímos a nossa base de dados com essa importante junção de dados de diferentes localidades. Em seguida, apresentamos as covariadas que foram construídas a partir dessa base de dados.

Enumeramos cada uma das covariadas justificando a sua importância para a análise e apresentado como foram construídas.

1. Distância de cada firma até o centro [DistSe]: conforme já mencionado antes, essa é uma covariada de tendência espacial onde queremos testar se há preferência espacial das

\footnotetext{
3.3 Disponível em: <https://sos.noaa.gov/datasets/nighttime-lights/> Acesso em: 06/10/2017

3.4 O uso de dados de iluminação noturna em modelos espaciais não é algo novo. Laurini (2016) já havia demonstrado a importância de efeitos aleatórios nos modelos que estimam a renda usando dados espaciais no caso do Brasil. O resultado desse estudo é base para a importância dessa covariada no modelo.
} 


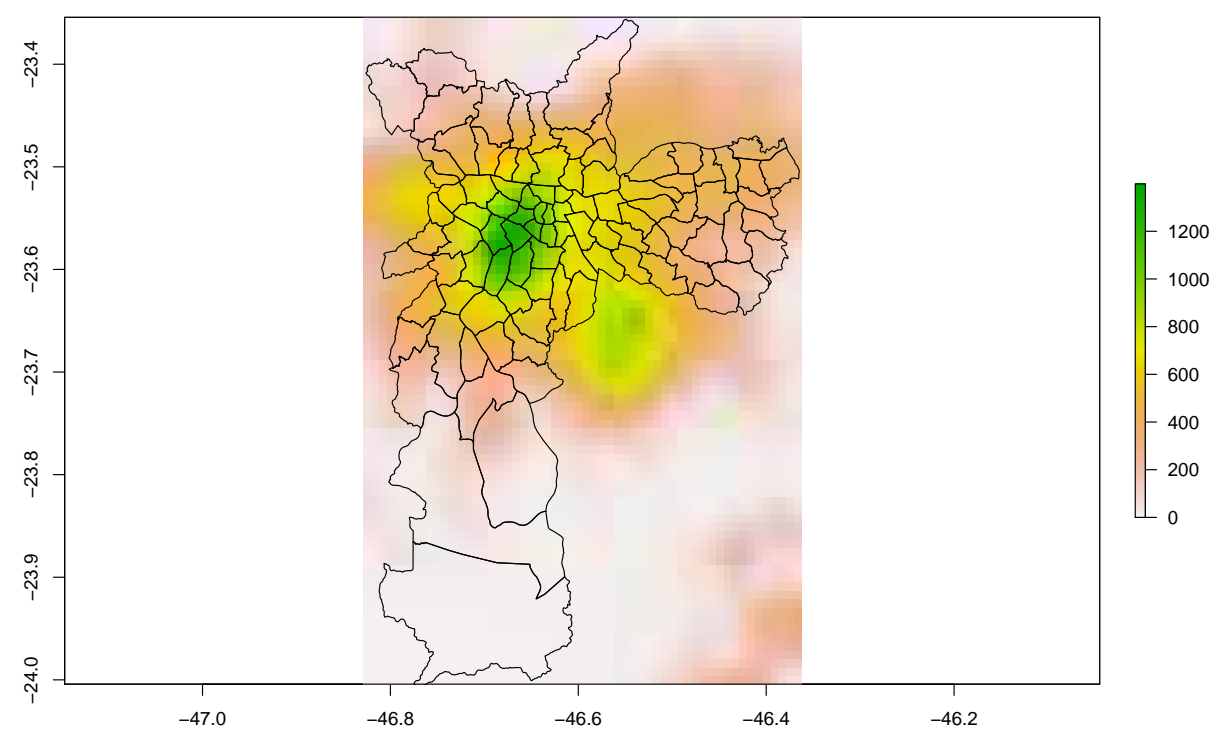

Figura 3.2 - Imagem de iluminação noturna da região de interesse.

empresas de serviço em se localizarem no centro da cidade. É comum ver em modelos espaciais parâmetros representando a distância até o centro dada a importância da geografia para indicadores econômicos, uma boa referência da importância desse parâmetro pode ser vista em Mills (1967). Vale mencionar que a escala dessa variável está em quilômetros e foi montada colocando a coordenada geográfica do "Marco da Sé"como ponto de referência para o cálculo da distância;

2. Luminosidade noturna [Illumination]: essa covariada, cuja intensidade de iluminação foi extraída por satélites, serve como proxy para infraestrutura e renda (Doll, Muller e Morley (2006), Laurini (2016) $)^{3.5}$. A motivação para a inclusão desta covariada no modelo é a relação entre crescimento econômico, renda e pobreza com luminosidade noturna, conforme explicado no famoso artigo de Henderson, Storeygard e Weil (2012) e em Elvidge et al. (2009). Assim, quer-se saber se a intensidade do processo aumenta em lugares onde há maior luminosidade. No entanto, o que se pretende saber é se regiões com alto índice de luminosidade influenciam na aglomeração de empresas. Isto é, saber se a luminosidade noturna servindo como proxy para infraestrutura e renda é um fator de atração para empresas, dado que regiões mais desenvolvidas podem ser atraentes para empresas. Essa covariada foi construída através de uma imagem raster que contém uma escala de cor para a distinguir a quantidade de luminosidade em cada região, conforme mostrado na Figura 3.2, que mostra a luminosidade noturna para o ano de 2000, sobreposta aos distritos do município de São Paulo.

$\overline{3.5}$ O uso da luminosidade noturna como um dado estatístico para estudos econômicos é rotineiro na literatura econômica: para uma análise mais detalhada deste procedimento: ver Chen e Nordhaus (2011). 


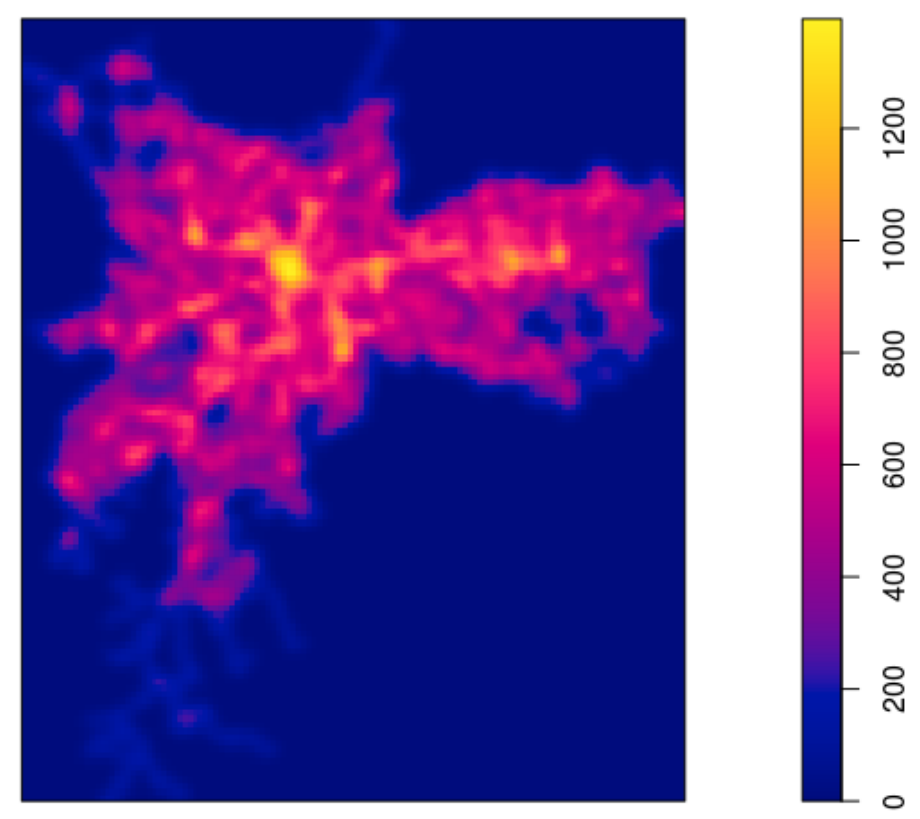

Figura 3.3 - Intensidade de linhas de ônibus calculada usando Kernel ajustado para as bordas.

3. Intensidade de linhas de ônibus [IntenBus]: o uso de técnicas SIG para estimação do acesso ao transporte público não é novo (O’Sullivan, Morrison e Shearer (2000)). Neste caso estamos usando a função de intensidade não paramétrica para obter uma medida sobre o acesso de linhas de ônibus em São Paulo, conforme mostrado na Figura 3.3. O uso de uma medida de intensidade, ao invés de uma medida de distância mais comum, é devido ao fato que os indivíduos têm acesso a diversas linhas de ônibus, e assim a variável de interesse é a disponibilidade dessas linhas ao invés da distância a linha mais próxima, que faz sentido quando essa linha concentra o acesso aos demais ramos de transporte, como acontece como o metrô e as linhas de trem. A inclusão dessa covariada se baseia na hipótese de que as empresas têm preferência por locais onde há sistema de transporte, diminuindo assim os custos relacionados. O impacto na economia urbana através da oferta de transporte público é estudado em termos de produtividade ou de localização espacial (Rietveld (1994)). A escala é dada pela função não paramétrica de intensidade;

4. Distância até a estação mais próxima de metrô e da CPTM [DistSub, DistTrain]: são duas covariadas. No modelo, a distância até o metrô é uma covariada e a distância até a CPTM é outra. Assim como as linhas de ônibus, essas duas covariadas também tem a intenção de testar a preferência das empresas por locais que dispõem de bons sistemas de transportes. A variável foi construída calculando a menor distância encontrada entre a mais próxima estação e a empresa. Da mesma forma que as demais distâncias, a unidade de medida são quilômetros;

5. Distância até a rodovia mais próxima [Dhighway]: essa covariada também está relacionada com a necessidade de diminuir custos de transportes. Existe uma relação conhecida 
entre desenvolvimento de cidades e a presença de autoestrada (Wheat (1969)). Empresas precisam de vias de escoamento de seus produtos e interligação entre sua localização e a região de moradia de seus funcionários, o que justifica a presença dessa covariada no modelo. O cálculo da distância também foi empregado para construir esta covariada, encontrando a menor distância entre a rodovia de cada empresa. A unidade também está em quilômetros;

6. Renda per-capita dos distritos [Income]: essa covariada visa testar se as empresas de serviços têm relação com a renda média per capita de cada distrito. Conforme visto nos principais modelos teóricos de localização industrial, presença de infraestrutura e necessidade de se estabelecer perto de seus mercados consumidores ou perto de mãode-obra qualificada é de interesse das empresas. Portanto, essa covariada pode ser um importante fator de atração industrial. Essa variável foi construída a partir dos dados do Censo demográfico, mas para encaixá-la no modelo foi preciso discretizar a janela do processo em sub-regiões com a menor agregação disponível, para as quais foram calculadas a renda média de cada sub-região com base nas informações censitárias (Censo 2000 do IBGE) pessoas que ali habitam. Feito isso, associamos a localização de cada empresa com a renda média da sub-região onde ela se encontra. A medição da variável é feita por renda per-capita em Reais para o ano de referência de 2000;

7. Densidade urbana [PopDensity: de maneira análoga com a covariada de renda média, quer-se testar se as empresas se localizam em regiões com maior densidade populacional. Essa variável foi construída para testar a relação das empresas com o tamanho do mercado local. Isto é, cidades com maior densidade populacional geram efeitos de aglomeração na produtividade da indústria, principalmente não manufatureira (Moomaw (1981)). A construção dessa covariada foi feita da mesma forma que a anterior, discretizando a janela do processo e calculando a densidade populacional de cada distrito censitário e relacionando-a com a localização de cada empresa.

8. Densidade de empresas de serviços [IntenFirms]: esta pesquisa está focada nas empresas que surgiram em 2000 em São Paulo, assim as empresas de serviço que já se encontravam anteriormente em São Paulo são base para essa covariada. Em linha com a covariada anterior sobre densidade urbana, o alvo dessa variável é analisar o efeito congestão das empresas em São Paulo dada a alta densidade urbana da região (Ciccone e Hall (1993)). Assim, a ideia subjacente desta covariada é que locais onde já se encontram muitas empresas semelhantes poderiam ser um fator de repulsão ou atração para novos negócios. Por efeito de congestão, novas empresas poderiam estar mais dispostas a se localizarem em locais com menor concorrência, se beneficiando até mesmo de um efeito de monopólio espacial ${ }^{3.6}$. Esta variável foi construída através da estimação não paramétrica

3.6 Ver, por exemplo, Beckmann e Thisse (1987). 


\section{Intensidade de Empresas até 1999}

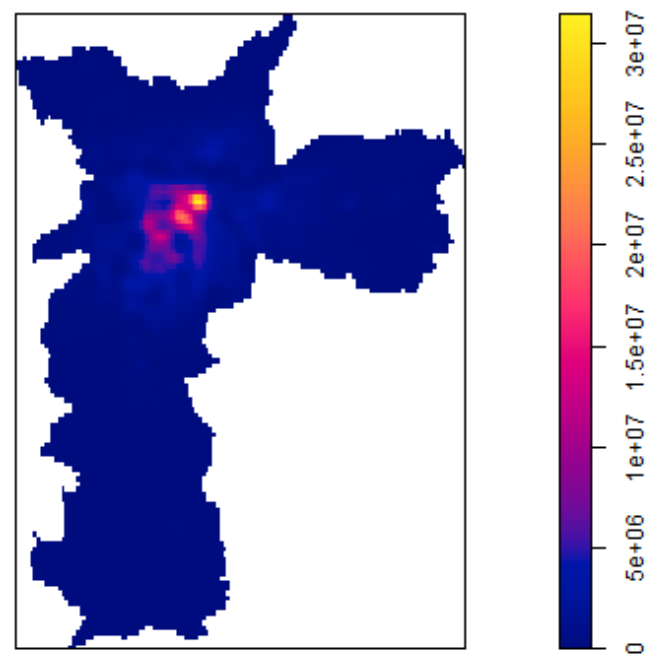

Figura 3.4 - Intensidade de empresas até o ano de 1999

da intensidade locacional das empresas existentes até o ano de 1999, e esta variável é mostrada na Figura 3.4. 


\section{Metodologia}

Separamos esta seção em 2 partes. A primeira parte descreve a construção e estimação do modelo de Poisson começando com uma breve introdução sobre processos pontuais. As principais fontes utilizadas para montar a explicação sobre processo pontual e processo de Poisson são, respectivamente, Baddeley, Bárány e Schneider (2007) e Baddeley, Rubak e Turner (2015).

A segunda parte faz uso dos conceitos já apresentados e explica o modelo LGCP, e estimação usando INLA. Utilizamos como fonte principalmente os conceitos de LGCP expostos em Møller, Syversveen e Waagepetersen (1998) e, para o INLA, utilizamos Gómez-Rubio e Palmí-Perales (2017) e Rue, Martino e Chopin (2009).

Ressaltamos também que a estimação de ambos os modelos será feita usando o R-INLA (Lindgren e Rue (2015)), o que permite comparação direta entre os modelos.

\subsection{Processos pontuais e o modelo de Poisson}

Um processo pontual é denotado por uma letra maiúscula como $\mathbf{X}$ ou $\mathbf{Y}$. Assim, definindo uma sub-regiões limitada qualquer como sendo $B$, estamos interessados no número $n(\mathbf{X} \cap \boldsymbol{B})$ de pontos do processo pontual $\mathbf{X}$ que caem na sub-região $B$, definidos no espaço bi-dimensional $\mathbb{R}^{2}$. Dessa forma, podemos afirmar que $n(\mathbf{X} \cap B)$ é uma variável aleatória bem definida de interesse no contexto de processos pontuais.

Em processo pontual, os dados podem apresentar uma estrutura homogênea, isto é, os pontos não possuem preferência por nenhuma localização espacial, ou não homogênea, na qual a densidade média é espacialmente variável. O primeiro caso, apesar de ter propriedades interessantes, é mais limitado no sentido em que fenômenos com essa estrutura são bem menos presentes na natureza. O segundo caso compõe a maioria dos trabalhos sobre o tema. Ainda assim, homogeneidade significa que o número $n(\mathbf{X} \cap B)$ de pontos aleatórios presentes na sub-regiões $B$ possui valor médio $E n(\mathbf{X} \cap B)=\lambda|B|$.

O processo de Poisson não homogêneo é caracterizado, não só pela variável aleatória $n(\mathbf{X} \cap B)$, mas também pelas três seguintes propriedades:

- (I) uma função de intensidade: o número esperado de pontos dentro da região $B$ é dado pela integral $\mu=\int_{B} \lambda(u) d u$, sendo $\lambda(u)$ a função intensidade sobre a região $B^{4.1}$.

- (II) independência: o espaço é dividido entre regiões não sobrepostas, as observações que se encontram dentro dele são independentes uma das outras.

4.1 Essa é a principal diferença entre o processo de Poisson homogêneo para o não homogêneo. Pois no primeiro caso $\lambda$ é uma constante. 
- (III) contagem de distribuição Poisson: a variável aleatória $n(\mathbf{X} \cap B)$ segue uma distribuição de probabilidade de Poisson.

Nosso maior interesse reside em estudar $\lambda(u)$, que é a função intensidade do processo. Sendo assim, vamos definir esta função e descrever suas características. Sendo $\mathbf{X}$ o processo pontual definido no espaço métrico $S=\mathbb{R}^{2}$, então:

$$
\nu(B)=E\left[N_{X}(B)\right], \quad B \subset S,
$$

define uma medida $\nu$ em $S$, chamada de medida de intensidade de $\mathbf{X}$, dado que $\nu(B)<\infty$ para todo compacto $B$. Esta definição deixa claro a relação da intensidade com o primeiro momento do processo pontual. A seguir, sendo $\nu$ a medida de intensidade do processo pontual $\mathbf{X}$ em $\mathbb{R}^{2}$, se esta medida satisfaz:

$$
\nu(B)=\int_{B} \lambda(u) d u
$$

para alguma função $\lambda$, então chamamos $\lambda$ de função intensidade de $\mathbf{X}$.

Explicado como se caracteriza o processo de Poisson não homogêneo e a função intensidade, destacamos os primeiros tratamentos que serão utilizados nos dados. Antes de explicitar o modelo apropriado, algumas análises prévias são desejadas até mesmo para dar mais confiabilidade ao modelos e às hipóteses. Assim, como análise de primeiro momento, podemos calcular de forma não paramétrica a função intensidade $\lambda(u)$. Para isso, usamos o estimador de Kernel uniformemente ajustado (Diggle (1985)) cuja fórmula é a seguinte:

$$
\widetilde{\lambda}^{(U)}(u)=\frac{1}{e(u)} \sum_{i=1}^{n} k\left(u-x_{1}\right)
$$

para qualquer localização u dentro da janela W, onde $k(u)$ é a função de Kernel e $e(u)=$ $\int_{W} k(u-\nu) d \nu$ é fator de correção do viés causado pelo efeito de borda.

O interessante nesse estudo é a transformação das informações contidas na janela do processo em um continuum, revelando as subregiões onde há maior concentração de empresas. Essa análise exploratória, embora importante fator de informações, não leva em conta outras variáveis. Por isso que o modelo paramétrico do processo de Poisson é importante, gerando informações das covariadas.

Todo processo de Poisson é inteiramente descrito por $\lambda(u)$. O modelo Poisson portanto é um simples modelo de intensidade, isto é, possui somente uma equação descrevendo $\lambda(u)$ em uma relação loglinear com as covariadas. Sua maior característica é assumir que os pontos aleatórios do processo pontual são condicionalmente independentes, o que está de acordo com a teoria em economia urbana que usa somente variáveis condicionantes como determinantes de localização. Portanto, o modelo se encaixa perfeitamente para esses dados não homogêneos, conforme explicitado anteriormente.

As quatro propriedades a seguir caracterizam o modelo de Poisson não homogêneo, algumas delas idênticas às que caracterizam o processo de Poisson não homogêneo: 
- (I) Contagem de Poisson: o número $n(\mathbf{X} \cap B)$ de pontos dentro da sub-regiões $B$ tem a distribuição Poisson.

- (II) Intensidade: o número $n(\mathbf{X} \cap B)$ de pontos na sub-regiões $B$ tem o valor esperado

$$
E[n(\mathbf{X} \cap B)]=\int_{B} \lambda(u) d u
$$

- (III) Independência: se $B_{1}, B_{2}, \ldots$ são subregiões disjuntas no espaço, então $n(\mathbf{X} \cap$ $\left.B_{1}\right), n\left(\mathbf{X} \cap B_{2}\right), \ldots$ são variáveis aleatórias independentes.

- (IV) Propriedade condicional: dado que $n(\mathbf{X} \cap B)=n$, então os $n$ pontos são independentes e identicamente distribuídos, com densidade de probabilidade comum

$$
f(u)=\frac{\lambda(u)}{I}
$$

onde $I=\int_{B} \lambda(u) d u$.

Seguimos agora com a definição do modelo. O modelo proposto para estudar a intensidade do processo é o loglinear aditivo onde as covariadas que queremos testar entram aumentando ou diminuindo a intensidade do processo. Sendo assim, a equação do modelo é a seguinte:

$$
\lambda(u)=\exp [\alpha+\beta \mathbf{Z Z}(u)]
$$

O objetivo dessa pesquisa é estimar os parâmetros desse modelo, onde $\alpha$ é o coeficiente independente, isto é, a intensidade do processo quando $Z(u) \approx 0$ e $\beta$ é o vetor de parâmetros correspondentes às covariadas $\mathbf{Z}(\mathrm{u})$.

O principal mecanismo de estimação utilizado para a estimação de processos de Poisson é a estimação por máxima pseudoverossimilhança, que é um método relativamente simples de estimação mas apresenta limitações importantes. O primeiro são limitações em relação ao número de observações. Amostras muito grandes levam a problemas de convergência numérica e uma grande demanda computacional. O método de máxima pseudoverossimilhança também não permite a estimação de modelos de Cox log Gaussianos com efeitos aleatórios espaciais, que é a especificação utilizada neste trabalho. Nesta pesquisa, para estimar o modelo de Poisson optou-se por utilizar aproximações de Laplace que serão explicadas adiante, que não sofre dessas limitações, e favorece a comparação entre modelos. Apresentamos brevemente esta possibilidade como referência.

O método de máxima pseudoverossimilhança foi proposto por Besag (1977), e é baseado no fato que a pseudoverossimilhança para um processo de Poisson é igual a sua verossimilhança com uma constante normalizadora. Este método serve como uma aproximação para a verossimilhança ao ignorar a dependência entre as observações. Portanto, depende da dependência entre os pontos para a aproximação ser adequada.

A função de máxima verossimilhança deste processo pode ser escrita como:

$$
L(\boldsymbol{\theta})=L(\boldsymbol{\theta} ; \boldsymbol{x})=\lambda_{\theta}\left(x_{1}\right) \lambda_{\theta}\left(x_{2}\right) \ldots \lambda_{\theta}\left(x_{n}\right) \exp \left(\int_{W}\left(1-\lambda_{\theta}(u)\right) d u\right)
$$


onde $x_{1}, \ldots, x_{n}$ são pontos de $\mathbf{x}$. Já a função de pseudo-máxima verossimilhança proposto por Besag (1975) é escrita da seguinte maneira:

$$
P L(\boldsymbol{\theta}, \boldsymbol{x})=\left(\prod_{x_{i} \in x} \lambda_{\theta}\left(x_{i}\right)\right) \exp \left[-\int_{W} \lambda_{\theta}(u) d u\right]
$$

O estimador MPLE (maximum pseudolikelihood estimator) é o valor de $\boldsymbol{\theta}$ que maximiza essa função.

\subsection{Modelo LGCP e a estimação por INLA}

Antes de apresentar as principais equações que compõem o modelo LGCP, enumeramos as principais vantagens de um modelo de processo de Cox log-Gaussiano, além de sua principal característica de conseguir capturar os efeitos espacialmente estruturados e não estrutuados ${ }^{4.2}$ :

- (I) A distribuição fica completamente caracterizada pela função de intensidade e a função de correlação de pares do processo de Cox;

- (II) maior facilidade em derivar propriedades estatísticas, tais como propriedades de ordens mais altas que podem ser expressas pela intensidade e pela função de correlação;

- (III) o processo gaussiano subjacente e a superfície de intensidade podem ser preditos através da realização de um LGCP observado dentro de uma janela de ocorrência do processo usando estimação bayesiana;

- (IV) não há problemas com efeitos de borda dado que a distribuição do LGCP restrita ao subconjunto limitado é conhecida.

A partir dessas características, em seguida descrevemos como é a estrutura do modelo LGCP univariado. Seguindo a estrutura definida em Møller, Syversveen e Waagepetersen (1998), seja $\mathbf{X}$ agora um subconjunto aleatório localmente finito no plano $\mathbb{R}^{2}$. Diz-se que $\mathbf{X}$ é um processo de Cox conduzido por um processo de intensidade aleatório $\Lambda=\left\{\Lambda(s): s \in \mathbb{R}^{2}\right\}$ se a distribuição condicional de $\mathbf{X}$, dado $\Lambda$, for um processo de Poisson com função de intensidade $\Lambda(\cdot)$. Além disso, o modelo que representa o processo de intensidade é log-gaussiano, equacionado da seguinte maneira:

$$
\Lambda(s)=\exp \{Y(s)\}
$$

onde $\mathrm{Y}=\left\{\mathrm{Y}(\mathrm{s}): \mathrm{s} \in \mathbb{R}^{2}\right\}$ é um campo Gaussiano em $s \in \mathbb{R}^{2}$. Dessa forma, a dependência entre os pontos é modelada através de uma variável comum latente Gaussiana Z(·). Fica fácil de notar através da equação 4.9 a estrutura logarítmica que caracteriza parte do nome desse modelo. 
Dada essa breve introdução sobre LGCP, para que seja possível a modelagem, alguns passos são necessários. Primeiro, a janela de ocorrência do processo, que chamaremos de $W$, precisa ser discretizada da seguinte maneira: dividimos a região em $n_{l i n h a} X n_{c o l}$ células $\left\{s_{i j}\right\}$ com mesma área $\left|s_{i j}\right|$, com $\mathrm{i}=1, \ldots, n_{\text {linha }}$ e $\mathrm{j}=1, \ldots, n_{c o l}$. Define-se assim $y_{i j}$ como sendo o número observado de pontos em cada célula $\left\{s_{i j}\right\}$. Em seguida, definimos que $y_{i j}$ está condicionado no campo latente $\eta_{i j}$ cujas contagens são independentes, ou seja:

$$
y_{i j} \mid \eta_{i j} \sim \operatorname{Poisson}\left(\left|s_{i j}\right| \exp \left(\eta_{i j}\right)\right) \text {. }
$$

A partir disso, pode-se modelar apropriadamente o campo latente de forma a incluir as covariadas:

$$
\eta_{i}=\alpha+\sum_{j=1}^{\eta_{\beta}} \beta_{j} z_{j i}+f_{i}^{(s)}+f_{i}^{(u)},
$$

onde $\alpha$ é o intercepto, $\beta_{j}$ são os parâmetros estimados das covariadas do modelo, $f_{i}^{(s)}$ é o efeito espacial estruturado e $f_{i}^{(u)}$ o efeito não estruturado.

Estruturado o modelo LGCP, segue-se para a estimação do modelo através do INLA ${ }^{4.3}$. Para isso, definimos o vetor de efeitos latentes $x=\left(\eta_{1}, \ldots, \eta_{n}, \alpha, \beta_{1}, \ldots\right)$. Rue, Martino e Chopin (2009) mostram como esse processo representa um GMRF. Definimos também $Q\left(\theta_{1}\right)$ como sendo a matriz de precisão do modelo, que em geral é bastante esparsa e depende do número de hiperparâmetros $\theta_{1}$. Essa matriz será importante no processo de estimação.

Podemos escrever a distribuição posterior dos efeitos latentes $x$ e do vetor de hiperparâmetros $\theta=\left(\theta_{1}, \theta_{2}\right)$ da seguinte forma:

$$
\pi(x, \theta \mid y)=\frac{\pi(y \mid x, \theta) \pi(x, \theta)}{\pi(y)} \propto \pi(y \mid x, \theta) \pi(x, \theta)
$$

onde a última parte da equação revela que podemos ignorar a constante normalizadora para os cálculos numéricos. Além disso, $\pi(y)$ é a verossimilhança marginal e $\pi(y \mid x, \theta)$ é a verossimilhança do modelo.

Essa equação acima só é possível por conta das observações em y serem independentes, dado $x$. Além disso, há o fato de que a sua distribuição depende do número de hiperparâmetros $\theta$.

A distribuição conjunta $\pi(\mathbf{x}, \theta)$ pode ser expressa como $\pi(\mathbf{x} \mid \theta) \pi(\theta)$, onde $\pi(\theta)$ é a priori dos parâmetros. Obtendo assim a seguinte equação:

$$
\pi(\mathbf{x} \mid \theta) \propto|\mathbf{Q}(\theta)|^{1 / 2} \exp \left\{-\frac{1}{2} \mathbf{x}^{T} \mathbf{Q}(\theta) \mathbf{x}\right\}
$$

dado que $\mathbf{x}$ é um GMRF.

Assumindo independência entre as observações $\left(y_{1}, \ldots, y_{n}\right)$ dado $\mathbf{x}$ e $\theta$, então:

$$
\pi(\mathbf{y} \mid \mathbf{x}, \theta)=\prod_{i \in I} \pi\left(y_{i} \mid \mathbf{x}, \theta\right)
$$

4.3 usamos neste trabalho a mesma estrutura explicativa do INLA que foi apresentada por Gómez-Rubio e PalmíPerales (2017) 
onde $I$ é um índice de números naturais $1 \mathrm{a} n$.

Portanto, a distribuição posterior dos efeitos latentes e dos parâmetros pode ser escrita da seguinte maneira:

$$
\begin{array}{r}
\pi(\mathbf{x}, \theta \mid y) \propto \pi(\theta)|\mathbf{Q}(\theta)|^{1 / 2} \exp \left\{-\frac{1}{2} \mathbf{x}^{T} \mathbf{Q}(\theta)(x)\right\} \prod_{i \in I} \pi\left(y_{i} \mid \mathbf{x}, \theta\right) \\
=\pi(\theta)|\mathbf{Q}(\theta)|^{1 / 2} \exp \left\{-\frac{1}{2} \mathbf{x}^{T} \mathbf{Q}(\theta)(x)+\sum_{i \in I} \log \left(\pi\left(y_{i} \mid \mathbf{x}, \theta\right)\right)\right\}
\end{array}
$$

Falta agora descrever as distribuições marginais posteriores. Com relação aos efeitos latentes, pode ser escrito da seguinte maneira:

$$
\pi\left(x_{i} \mid \mathbf{y}\right)=\int \pi(\theta \mid \mathbf{y}) \pi(\theta \mid \mathbf{y}) d \theta
$$

já a distribuição marginal posterior dos hiperparâmetros $\theta_{i}$ é:

$$
\pi\left(\theta_{i} \mid \mathbf{y}\right)=\int \pi(\theta \mid \mathbf{y}) d \theta_{-i}
$$

onde $\theta_{-i}$ é o vetor de parâmetros $\theta$ exceto $\theta_{i}$.

A aproximação sugerida por Rue et al. (2008) é sugerida com base na aproximação $\tilde{\pi}(\theta \mid \mathbf{y})$ da marginal posterior de $\theta$ :

$$
\left.\tilde{\pi}(\theta \mid \mathbf{y}) \propto \frac{\pi(\mathbf{x}, \theta, \mathbf{y})}{\tilde{\pi}_{\mathbf{G}}(\mathbf{x} \mid \theta, \mathbf{y})}\right|_{\mathbf{x}=\mathbf{x}^{*}(\theta)}
$$

em que $\tilde{\pi}_{\mathbf{G}}(\mathbf{x} \mid \theta, \mathbf{y})$ é a aproximação Gaussiana da condicional completa de $\mathbf{x}$ e $\mathbf{x}^{*}$ é a moda da condicional completa de $\mathbf{x}$, para um dado $\theta$.

A partir deste ponto, Rue, Martino e Chopin (2009) descrevem em alguns passos como computar o INLA e também três diferentes aproximações numéricas, i.e. a Gaussiana, a de Laplace e uma aproximação simplificada de Laplace. Os detalhes técnicos dessa aproximação não compõem o escopo do trabalho, para mais detalhes computacionais basta consultar o artigo na íntegra dos autores citados ou Blangiardo e Cameletti (2015).

Para aplicar o INLA no modelo LGCP, é preciso definir alguns detalhes do modelo como o valor inicial dos hiperparâmetros. A especificação da priori dos efeitos espaciais escolhida é o modelo autorregressivo CAR ("Conditional Autoregressive") definida originalmente por Besag (1974), e que pode ser facilmente implantada através do R-INLA. O modelo de Besag para um vetor aleatório qualquer $\mathbf{x}=\left(x_{1}, \ldots, x_{n}\right)$ é definido como:

$$
x_{i} \mid x_{j}, i \neq j, \tau \sim \mathcal{N}\left(\frac{1}{n_{i}} \sum_{i \sim j} x_{j}, \frac{1}{n_{i} \tau}\right)
$$

onde $n_{i}$ é o número de vizinhos da região $i, i \sim j$ indica que as regiões $i$ e $j$ são vizinhas. Neste procedimento, os vizinhos são construídos na discretização do processo de estimação.

Para usar o modelo de Besag nos hiperparâmetros do modelo LGCP, é necessário definir o parâmetro de precisão $\tau$ representado da seguinte maneira:

$$
\theta_{1}=\log \tau
$$


Neste trabalho, a matriz de precisão será definida como $\tau \mathbf{Q}$, onde $\mathbf{Q}$ é:

$$
\mathbf{Q}_{i j}= \begin{cases}n_{i} & i=j \\ -1 & i \sim j \quad 1 \leq i, j \leq n \\ 0 & \text { caso contrário }\end{cases}
$$

o modelo soma zero para que seja identificado. Da mesma forma que antes, o termo $n_{i}$ é o número de vizinhos da região i, já $i \sim j$ indica que $i$ e $j$ são vizinhos. O chute inicial concedido foi $1 / \operatorname{var}\left(y_{i}\right)$, onde $y_{i}$ são as observações. 



\section{Aplicação Empírica}

Nesta seção, apresentamos os resultados da estimativa dos modelos Poisson e LGCP para a localização das empresas e interpretamos os resultados à luz da teoria da economia urbana, discutindo limitações e respostas encontradas.

O primeiro resultado a ser mostrado é a estimativa não paramétrica para a intensidade observada das empresas do setor de serviços, estimado usando kernel ajustado para viés de borda (e.g. (BADDELEY; RUBAK; TURNER, 2015)). O resultado é mostrado na figura 5.1:

Esta figura mostra a intensidade estimada não parametricamente na janela do processo. A escala de cores demonstra claramente que no centro de São Paulo ocorre uma maior intensidade do processo, isto é, possui uma maior concentração de empresas de serviços. Isso está de acordo com a teoria de aglomeração e com o que foi observado na Figura 3.1. No entanto, esta estimação não paramétrica não leva em consideração a influência de covariadas. Sendo assim, não é possível estimar o impacto dos fatores condicionantes e de atração e repulsão de empresas, somente afirmar onde há maior e menor intensidade, sendo apenas uma estatística descritiva de referência para o processo de intensidade.

Para obter uma estimativa do impacto das covariadas sobre o padrão de localização, estimamos inicialmente um modelo de Poisson não-homogêneo utilizando o conjunto de variáveis descrito na Seção 3. O grid utilizado para a estimação dos processos pontuais corresponde a

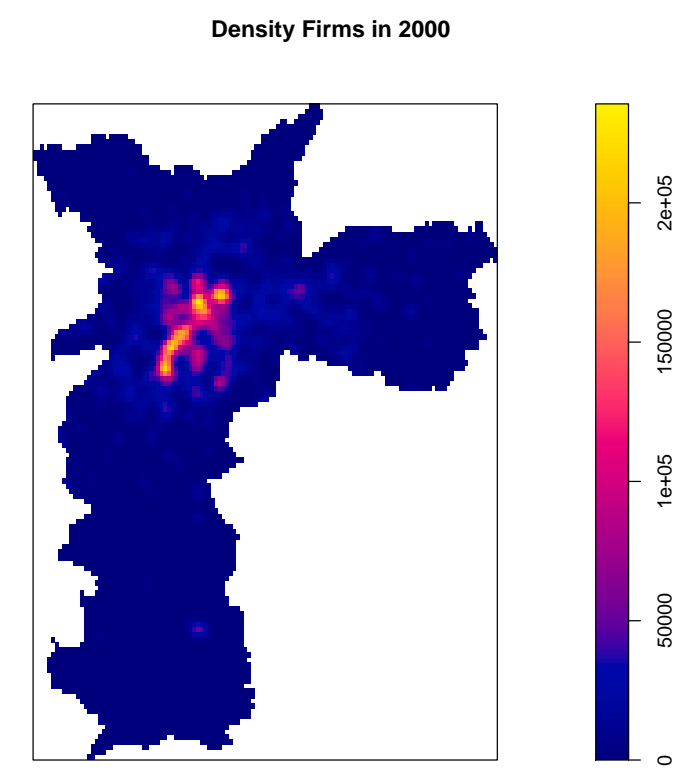

Figura 5.1 - Estimativa da intensidade não-paramétrica da localização das novas empresas no ano 2000, usando Kernel ajustado para efeitos de borda. 


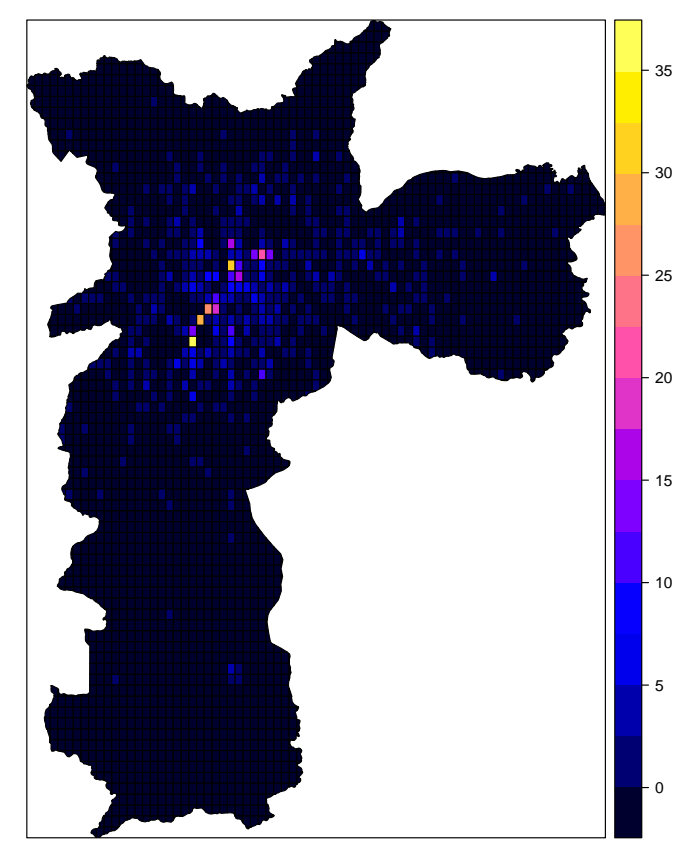

Figura 5.2 - Contagem de firmas no grid utilizado na estimação.

um conjunto de 2776 retângulos com tamanho médio de $0.5453 \mathrm{~km} 2$ em cada retângulo. Esta escolha de grid permite uma aproximação adequada para o continuum espacial, e assim uma aproximação computacional com elevada precisão para o processo espacial de interesse. A Figura 5.2 mostra a contagem de empresas no grid de estimação utilizado na estimação dos processos pontuais modelados nesse trabalho. A Tabela 5.1 mostra as estatísticas descritivas para as variáveis utilizadas no estudo, para o grid construído para a estimação.

\begin{tabular}{rrrrr}
\hline & Média & E. P. & max & min \\
\hline Contagem de firma & 0.3850865 & 1.7035574 & 35 & 0 \\
DistSe & 21.093 & 12.285 & 52.183 & 0.310 \\
Dmetro & 12.680 & 10.032 & 38.835 & 0.096 \\
DistTrains & 9.640 & 9.720 & 36.962 & 0.092 \\
Illumination & 430.186 & 265.800 & 1398.600 & 23.528 \\
PopDensity & 7553.269 & 10263.493 & 63297.093 & 0.643 \\
Dhighway & 5.621 & 4.212 & 20.518 & 0.000 \\
IntenBus & 332.173 & 264.970 & 1384.976 & 0.000 \\
Income & 1095.490 & 1517.744 & 16943.921 & 106.065 \\
IntenFirms & $7.378724 \mathrm{e}+05$ & $1.912715 \mathrm{e}+06$ & $2.655444 \mathrm{e}+07$ & 0 \\
Tamanho do Grid & 2776 & & & \\
\hline
\end{tabular}

Tabela 5.1 - Estatística Descritiva - Variáveis no Grid Estimado

A Tabela 5.2 mostra os resultados obtidos para a distribuição posterior dos parâmetros de interesse, usando a estimação por aproximações integradas aninhadas de Laplace (INLA). Note que nessa estimação os parâmetros são referentes a log-intensidade do processo, sendo o uso do log um mecanismo para impor a positividade na estrutura condicional de intensidade do 
Tabela 5.2 - Estimação dos parâmetros do modelo de Poisson

\begin{tabular}{lcccccc}
\hline & \multicolumn{7}{c}{ Parâmetro } \\
\cline { 2 - 7 } Variável & Média & E. P. & $0.025 \mathrm{q}$ & $0.5 \mathrm{q}$ & $0.975 \mathrm{q}$ & moda \\
\hline (Intercepto) & -2.63 & 0.267 & -3.16 & -2.63 & -2.11 & -2.63 \\
DistSe & 0.0512 & 0.013 & 0.0256 & 0.0513 & 0.0765 & 0.0514 \\
DistSub & -0.0944 & 0.0134 & -0.121 & -0.0944 & -0.068 & -0.0944 \\
DistTrain & -0.0333 & 0.0155 & -0.0642 & -0.0332 & -0.00344 & -0.0328 \\
Dillumin & 0.00315 & 0.000201 & 0.00276 & 0.00315 & 0.00355 & 0.00315 \\
PopDensity & $-4.04 \mathrm{e}-06$ & $3.31 \mathrm{e}-06$ & $-1.07 \mathrm{e}-05$ & $-4 \mathrm{e}-06$ & $2.35 \mathrm{e}-06$ & $-3.93 \mathrm{e}-06$ \\
Dhighway & 0.00933 & 0.0163 & -0.0227 & 0.00935 & 0.0412 & 0.0094 \\
IntenBus & 0.000938 & 0.000201 & 0.000544 & 0.000938 & 0.00133 & 0.000938 \\
Income & $-4.26 \mathrm{e}-05$ & $1.72 \mathrm{e}-05$ & $-7.7 \mathrm{e}-05$ & $-4.24 \mathrm{e}-05$ & $-9.59 \mathrm{e}-06$ & $-4.19 \mathrm{e}-05$ \\
IntenFirms & $6.07 \mathrm{e}-08$ & $7.99 \mathrm{e}-09$ & $4.49 \mathrm{e}-08$ & $6.08 \mathrm{e}-08$ & $7.63 \mathrm{e}-08$ & $6.08 \mathrm{e}-08$ \\
DIC & 3325.76 & & & & & \\
Moran I & 0.0552950625 & $\mathrm{p}-\mathrm{valor}$ & $1.619 \mathrm{e}-08$ & & & \\
\hline
\end{tabular}

processo.

A tabela 5.2 mostra a estimativa da média posterior de cada parâmetro na primeira coluna, o erro padrão, os intervalos de credibilidade e a moda para cada parâmetro estimado. As últimas linhas da Tabela também relatam o DIC (Deviance Information Criteria) e um teste de correlação espacial nos resíduos, usando uma estatística Moran I modificada. A intensidade do processo de ponto quando as covariáveis são iguais a zero é próxima da exponencial de $-2,63$, o que equivaleria a um valor médio de 0,07207846 novas empresas por cada $\mathrm{km} 2$, na ausência de outros valores de condicionamento.

É possível observar que, exceto pela distância da rodovia mais próxima (Dhighway); Todas as outras covariáveis são relevantes no modelo de Poisson. Note-se que a magnitude dos efeitos refere-se à intensidade do log para a janela de estimativa medida em km2 e, portanto, a magnitude dos coeficientes é proporcional a esse efeito.

De acordo com este primeiro modelo, o coeficiente de iluminação noturna, a intensidade das linhas de ônibus e o número de empresas na região seguem como fatores fixos de atração das empresas. A maioria dessas covariadas está fortemente vinculada à questão da infraestrutura da cidade. A distância do centro é positiva nesta especificação, o que pode indicar um fator de saturação de mercado em empresas de serviços na região central de São Paulo.

Outro ponto importante é notar que alguns resultados são negativos, como a distância da estação de metrô mais próxima e a distância até a estação de trem mais próxima. Isso pode indicar que esses fatores repelem as empresas. A hipótese do primeiro efeito é que as regiões próximas das estações de metrô são mais caras, o que pode decompor a proximidade ao equilíbrio espacial. A covariância da densidade firme (IntenFirms) é positiva. Isso pode significar que muitas empresas presentes em uma região não diminuem a presença de novas empresas por causa do congestionamento em um ambiente de mercado competitivo.

As figuras 5.3 e 5.4 mostram o ajuste do modelo com os parâmetros estimados e o erro de estimativa. Podemos observar que o modelo de Poisson reproduz o padrão de concentração espacial geral observado nos dados (Figura 5.3), mas não pode adequadamente ajustar a variabilidade 


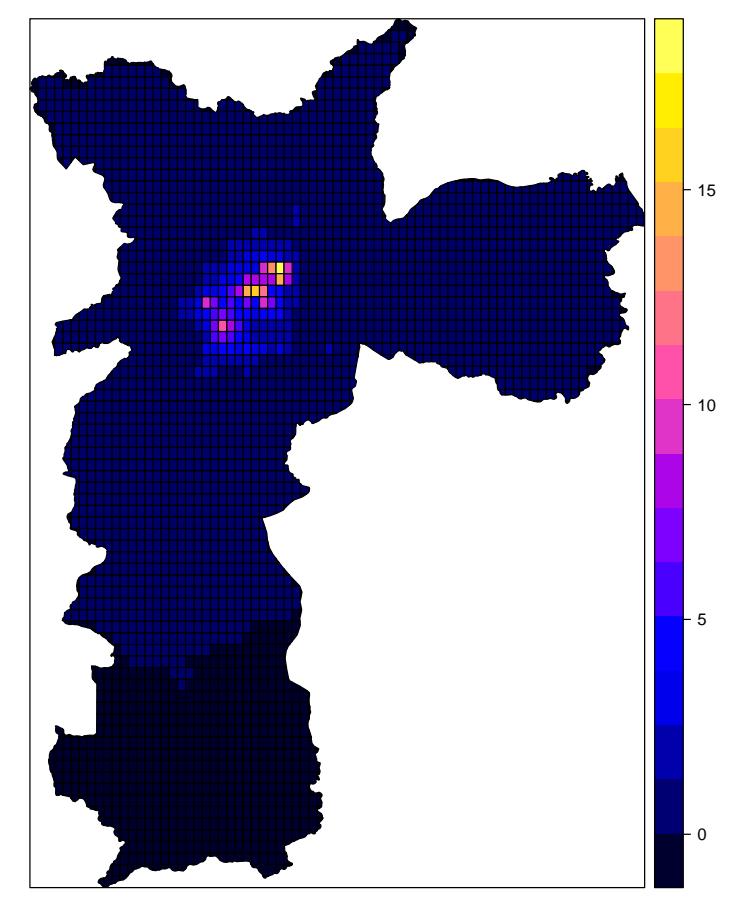

Figura 5.3 - Ajuste do modelo de Poisson irrestrito no município de São Paulo.

na localização espacial das empresas do setor de serviços. Este resultado é bastante evidente na Figura 5.4 com os resíduos (diferença entre a contagem esperada e a contagem observada em cada célula da grade). Pode-se observar que o modelo de Poisson deixa um padrão claro de concentração espacial nos resíduos de contagem, bem como várias células com erros de ajuste bastante consideráveis. Este resultado de dependência espacial nos resíduos também é confirmado pelo resultado do teste de correlação espacial em resíduos (o Ajuste do I de Moran para dados discretos) relatado na Tabela 5.2, que mostra um p-valor próximo de zero, indicando um padrão relevante de dependência espacial dos resíduos do modelo.

Esses problemas indicam que o modelo Poisson não consegue explicar adequadamente o processo de localização espacial das empresas do setor de serviços, mesmo com o conjunto completo de covariadas. Assim existe algum conjunto de variáveis não incluídas no modelo ou processos de atração/repulsão omitidos nesta especificação. Essas limitações motivam o uso do modelo LGCP, que permite incluir um processo de intensidade estocástica, em contraste com a intensidade determinística assumida no processo de Poisson, e também possibilitam a inclusão de uma estrutura probabilística para a aproximação de processos de clustering ou repulsão.

Como discutido na seção de metodologia, para realizar a estimação do processo LGCP controlando para possíveis efeitos de dependência espacial não observada, nós utilizamos uma especificação que modela a log intensidade do processo pontual como a soma dos efeito condicional das variáveis explicativas no modelo (partindo do mesmo conjunto utilizado para o processo de Poisson), mais um processo de dependência espacial, modelado como um processo CAR de efeitos aleatórios espaciais, e um processo de efeito aleatório espacial não estruturado.

As tabelas 5.3 e 5.4 apresentam o resultado da estimativa do modelo LGCP. Os parâmetros 


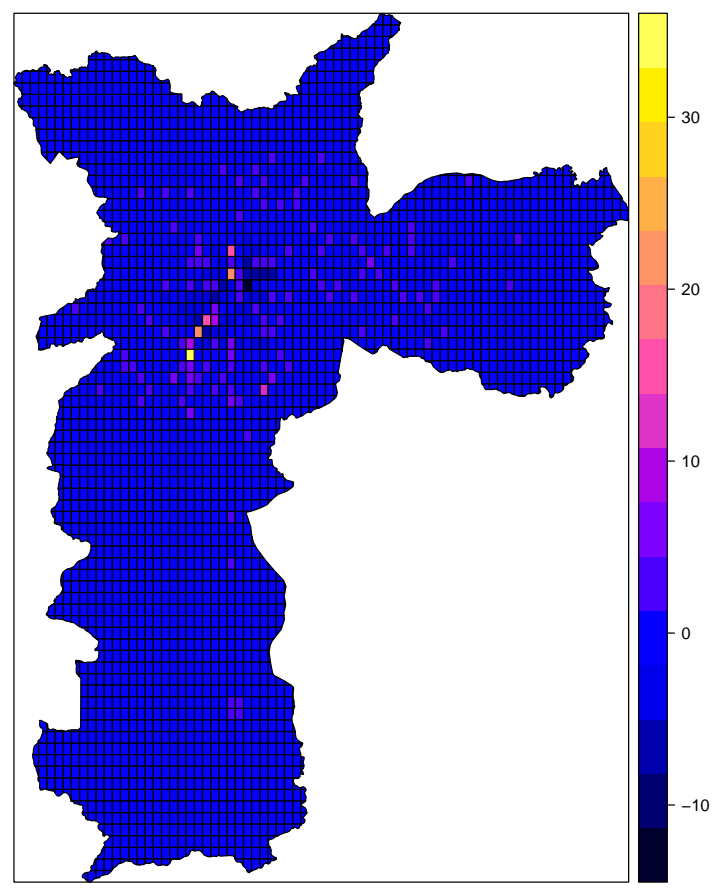

Figura 5.4 - Erro do modelo de Poisson irrestrito no município de São Paulo.

Tabela 5.3 - Estimação dos parâmetros do modelo LGCP irrestrito

\begin{tabular}{lcccccc}
\hline & \multicolumn{7}{c}{ Parâmetro } \\
\cline { 2 - 7 } Variável & Mean & Std. error & $0.025 \mathrm{q}$ & $0.5 \mathrm{q}$ & $0.975 \mathrm{q}$ & mode \\
\hline Intercepto) & -1.27 & 1.07 & -3.35 & -1.29 & 0.867 & -1.31 \\
DistSe & -0.0861 & 0.0671 & -0.22 & -0.0856 & 0.0449 & -0.0844 \\
DistSub & -0.046 & 0.057 & -0.159 & -0.0459 & 0.0662 & -0.0458 \\
DistTrain & 0.0154 & 0.0511 & -0.087 & 0.016 & 0.114 & 0.0172 \\
Illumination & 0.00166 & 0.000769 & 0.000128 & 0.00166 & 0.00316 & 0.00167 \\
PopDensity & $1.38 \mathrm{e}-06$ & $5.23 \mathrm{e}-06$ & $-9.03 \mathrm{e}-06$ & $1.43 \mathrm{e}-06$ & $1.15 \mathrm{e}-05$ & $1.53 \mathrm{e}-06$ \\
Dhighway & 0.00129 & 0.0473 & -0.0928 & 0.00157 & 0.0938 & 0.00216 \\
IntenBus & 0.00103 & 0.000402 & 0.000238 & 0.00102 & 0.00182 & 0.00102 \\
Income & $-4.43 \mathrm{e}-05$ & $3.22 \mathrm{e}-05$ & -0.000108 & $-4.41 \mathrm{e}-05$ & $1.84 \mathrm{e}-05$ & $-4.38 \mathrm{e}-05$ \\
IntenFirms & $1.41 \mathrm{e}-07$ & $2.85 \mathrm{e}-08$ & $8.6 \mathrm{e}-08$ & $1.41 \mathrm{e}-07$ & $1.98 \mathrm{e}-07$ & $1.4 \mathrm{e}-07$ \\
DIC & 2519.68 & & & & & \\
Moran I & -0.0401519946 & $\mathrm{p}$-valor & 1 & & & \\
\hline
\end{tabular}

Tabela 5.4 - Estimação dos hiperparâmetros dos efeitos aleatórios do modelo LGCP irrestrito

\begin{tabular}{lcccccc}
\hline & \multicolumn{6}{c}{ Parâmetro } \\
\cline { 2 - 7 } Variável & Média & E. P. & $0.025 \mathrm{q}$ & $0.5 \mathrm{q}$ & $0.975 \mathrm{q}$ & moda \\
\hline Precisão Espacial & 0.8 & 0.21 & 0.444 & 0.783 & 1.26 & 0.75 \\
Precisão não estruturada & 2.53 & 0.871 & 1.33 & 2.36 & 4.7 & 2.06 \\
\hline
\end{tabular}


relacionados à covariada (Tabela 5.3) indicam padrões semelhantes aos obtidos pelo modelo de Poisson, como o efeito negativo da distância para a renda central, subterrânea e per capita e os efeitos positivos da densidade da linha de ônibus, a iluminação noturna e densidade das empresas existentes. Mas agora a distância ao centro, metro e trens e a densidade urbana incluem o zero no intervalo de credibilidade, indicando um possível efeito nulo na intensidade do log. Uma possível interpretação é a presença de efeito de confounding entre essas variáveis e o efeito espacial latente, indicando que esse efeito agora captura parte do impacto dessas variáveis na localização de novas empresas.

No entanto, a inclusão de efeitos aleatórios na intensidade permite um ajuste muito mais preciso para a log intensidade. Podemos observar que o DIC melhorou muito em relação ao modelo de Poisson, passando de 3325,76 para 2519,68. Esse ganho de ajuste penalizado pode ser visto na Figura 5.5, mostrando o ajuste do modelo. A figura mostra que o modelo LGCP pode capturar com mais precisão o padrão de concentração observado na região central da cidade, mas também as demais fontes de concentração de empresas fora do eixo central observadas na cidade de São Paulo, reproduzindo a heterogeneidade espacial na região com a dispersão de empresas.

Esse ganho de poder explicativo vem da inclusão do efeito espacial estruturado através da estrutura CAR. Podemos observar na Tabela 5.4 que o componente de precisão relacionado a este efeito é bastante relevante, em contraste com o efeito não estruturado que apresenta uma maior precisão, ou seja, indicando menor poder explicativo. Na Figura 5.6 vemos o mapa com o efeito aleatório espacial estimado (média posterior deste efeito), que indica a existência de um padrão de agrupamento latente não capturado pelas variáveis explicativas do modelo, uma vez que existe uma dependência espacial evidente em os efeitos capturados por este efeito, sendo especialmente importante para explicar o padrão de localização nas regiões mais distantes do centro. Note que esse efeito pode ser interpretado como uma aproximação para os efeitos de agrupamento, ou também como uma forma de agregar todas as variáveis relevantes que possuem um padrão espacial que estão omitidas no modelo. A importância desse efeito de dependência espacial sobre o efeito aleatório não estruturado também pode ser interpretada a partir da Figura 5.7, que mostra a projeção espacial dos efeitos aleatórios não estruturados. Podemos observar que a magnitude desses efeitos é muito pequena, indicando que a principal fonte de variação estocástica na intensidade do log é explicada pelo componente da dependência espacial latente.

O erro de ajuste obtido pelo modelo LCGP é mostrado na Figura 5.8. É possível notar que neste componente não há padrões sistemáticos de dependência (não existe uma concentração clara de erros positivos ou negativos) e a magnitude desse efeito é muito menor que a observada pelos erros obtidos pelo modelo de Poisson (Figura 5.4). Observe que esta evidência é suportada pelo resultado do teste de dependência espacial relatado na última linha da Tabela 5.3, cujo p-valor de 1 suporta a hipótese de não haver dependência espacial nos resíduos do modelo, validando a especificação proposta. Esses resultados indicam que o modelo LGCP pode capturar mais precisamente os componentes observados e latentes que determinam a localização espacial das empresas de serviços na cidade de São Paulo. 


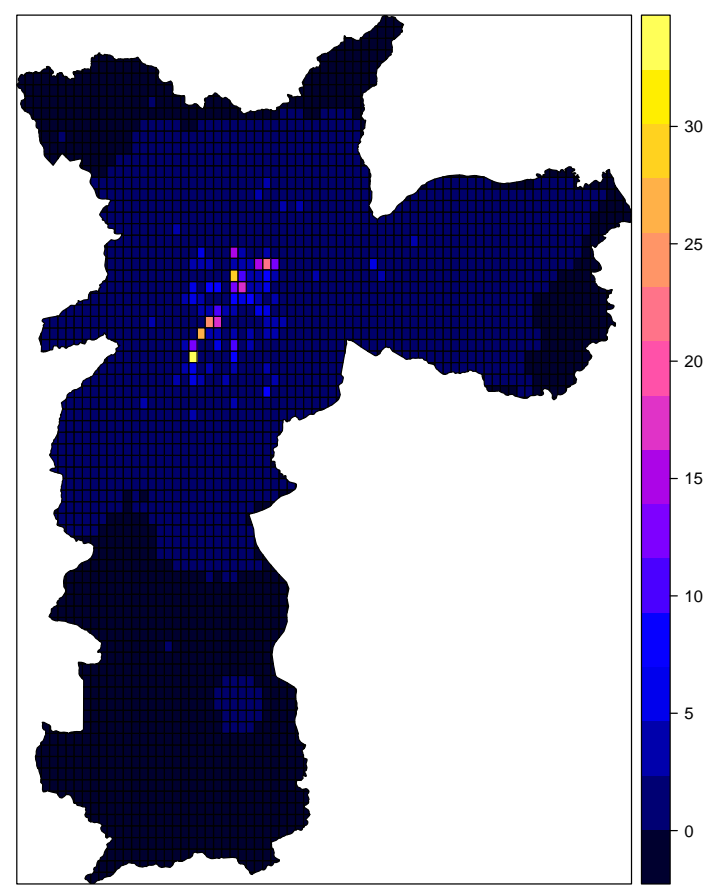

Figura 5.5 - Ajuste - LGCP irrestrito.

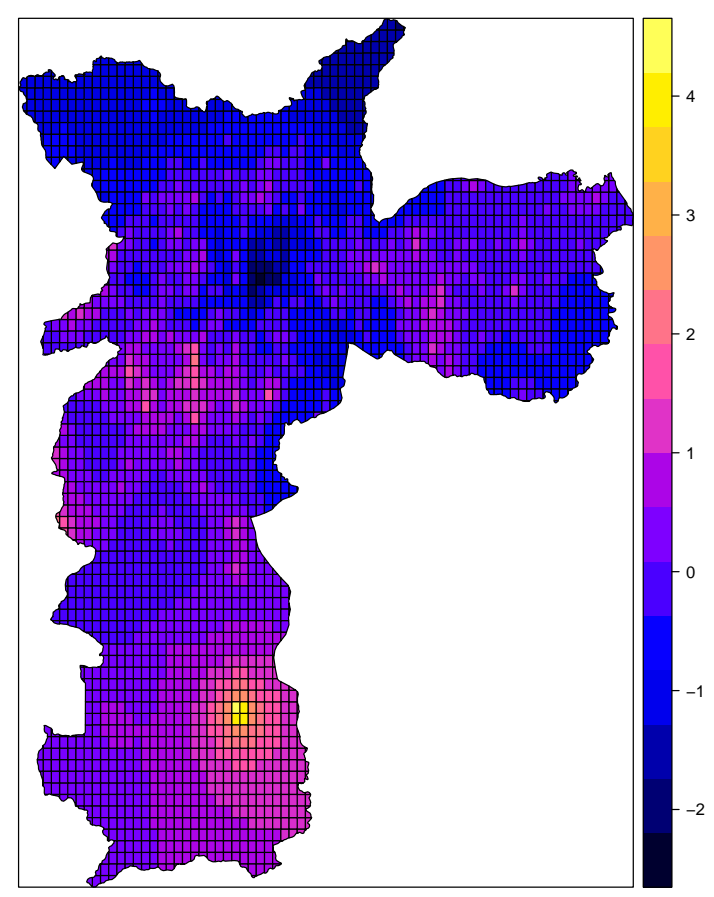

Figura 5.6 - Efeito estruturado capturado pelo modelo LGCP irrestrito. 


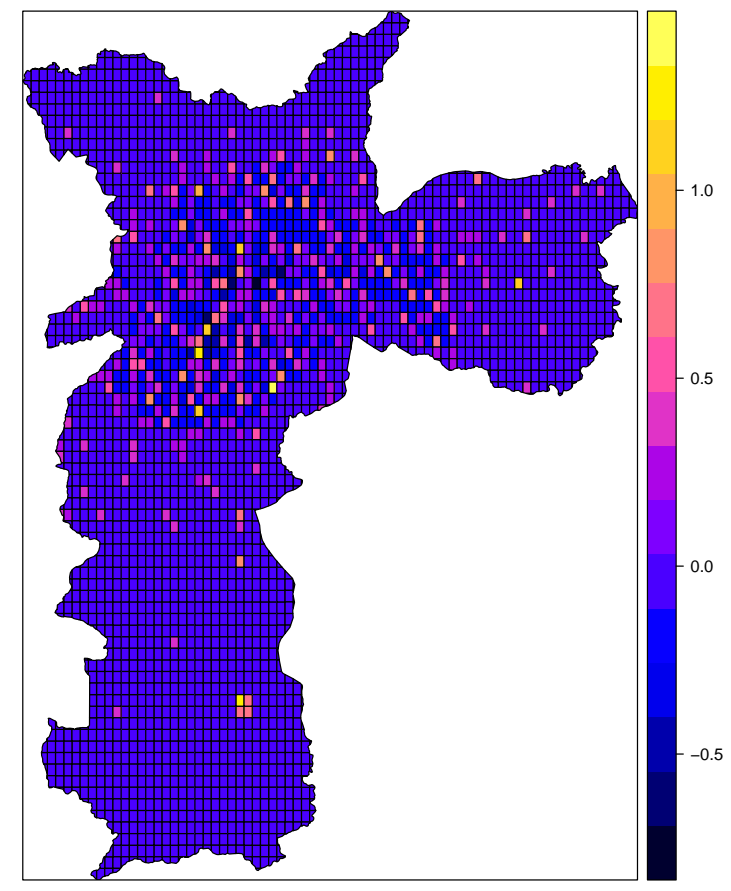

Figura 5.7 - Efeito não estruturado capturado pelo modelo LGCP irrestrito.

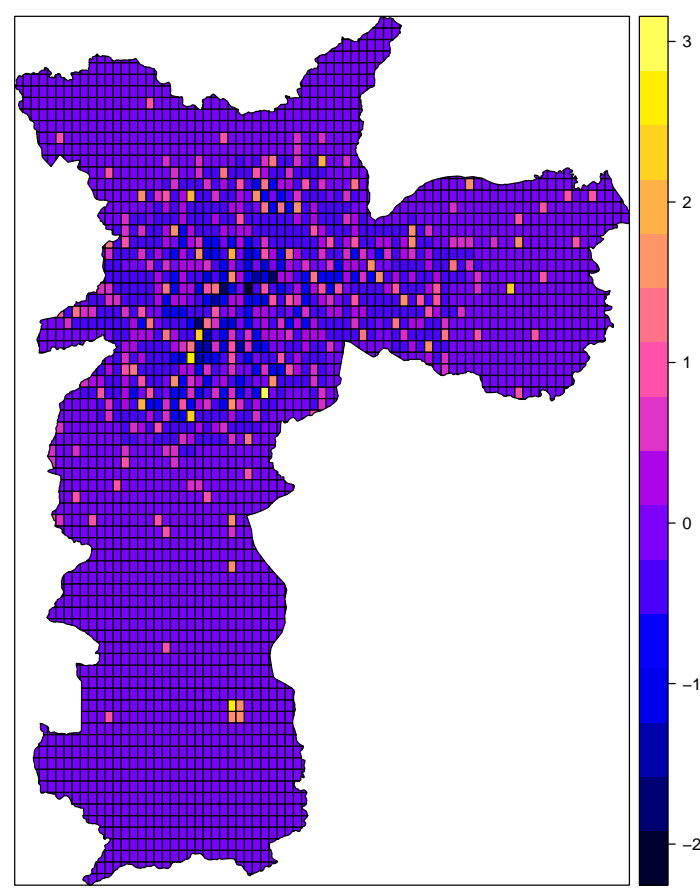

Figura 5.8 - Erro do modelo LGCP irrestrito: predito menos observado. 
Tabela 5.5 - Estimação dos parâmetros do modelo LGCP restrito

\begin{tabular}{lcccccc}
\hline & \multicolumn{7}{c}{ Parâmetro } \\
\cline { 2 - 7 } Variável & Média & E. P & $0.025 \mathrm{q}$ & $0.5 \mathrm{q}$ & $0.975 \mathrm{q}$ & moda \\
\hline Intercept & 1.15 & 0.306 & 0.546 & 1.15 & 1.75 & 1.15 \\
DistSe & -0.21 & 0.0448 & -0.299 & -0.21 & -0.123 & -0.21 \\
DistSub & -0.0063 & 0.0568 & -0.119 & -0.00604 & 0.105 & -0.00552 \\
DistTrain & 0.0233 & 0.0525 & -0.0817 & 0.0238 & 0.125 & 0.0248 \\
Illumination & 0.000195 & 0.000582 & -0.000954 & 0.000196 & 0.00134 & 0.000199 \\
IntenBus & 0.000976 & 0.000386 & 0.000224 & 0.000974 & 0.00174 & 0.00097 \\
IntenFirms & $1.5 \mathrm{e}-07$ & $2.83 \mathrm{e}-08$ & $9.55 \mathrm{e}-08$ & $1.5 \mathrm{e}-07$ & $2.06 \mathrm{e}-07$ & $1.5 \mathrm{e}-07$ \\
DIC & 2517.44 & & & & & \\
Moran I & $-4.921566 \mathrm{e}-02$ & p-valor & 1 & & & \\
\hline
\end{tabular}

Tabela 5.6 - Estimação dos hiperparâmetros dos efeitos aleatórios do modelo LGCP restrito

\begin{tabular}{lcccccc}
\hline & \multicolumn{7}{c}{ Parâmetro } \\
\cline { 2 - 7 } Variável & Média & E.P. & $0.025 \mathrm{q}$ & $0.5 \mathrm{q}$ & $0.975 \mathrm{q}$ & moda \\
\hline Precisão Espacial & 0.718 & 0.172 & 0.417 & 0.708 & 1.08 & 0.692 \\
Precisão não estruturada & 2.86 & 1.08 & 1.46 & 2.61 & 5.6 & 2.21 \\
\hline
\end{tabular}

Para verificar se é possível eliminar alguma variável não relevante do modelo, procedemos a um processo de seleção do modelo, usando como critério o DIC. A especificação selecionada DIC é relatada nas tabelas 5.5 e 5.6, e consiste em retirar as variáveis de densidade populacional (PopDensity), distância das rodovias (Dhighway) e renda per capita. As duas primeiras variáveis incluíram zero no intervalo de credibilidade na especificação completa e a retirada de renda pode ser interpretada pelo controle dessa variável pela presença de iluminação noturna no modelo, que pode servir como proxy para a renda. No entanto, o ganho no DIC (2519.68 a 2517.44) é marginal, indicando que os dois modelos são basicamente equivalentes em termos de ajuste do modelo penalizado.

Podemos observar que o poder explicativo desta especificação alternativa é bastante semelhante ao modelo completo, como pode ser observado ao ajustar este modelo mostrado na Figura 5.9. Também é possível notar que os efeitos aleatórios também são bastante semelhantes nesta especificação, como pode ser visto nas Figuras 5.10 e 5.11, respectivamente, contendo efeito espacial e efeito não estruturado. Os resíduos do modelo (Figura 5.12) também são bastante semelhantes, indicando a equivalência observacional dessas duas especificações. O resultado da ausência de dependência espacial, indicado pelo p-valor 1 do teste de I-Moran modificado, também indica que esta especificação pode capturar adequadamente o processo de localização espacial de empresas da cidade de São Paulo.

Para quantificar as diferenças preditivas entre as especificações, realizamos uma análise de ajuste comparando os valores observados das contagens observadas em cada célula na discretização do espaço com as previsões de cada modelo. A Tabela 5.7 mostra o erro médio (ME), o erro quadrático médio (RMSE), o erro médio absoluto (MAE), o erro médio porcentual (MPE) e o erro médio porcentual absoluta (MAPE) obtido nas diferentes especificações. 


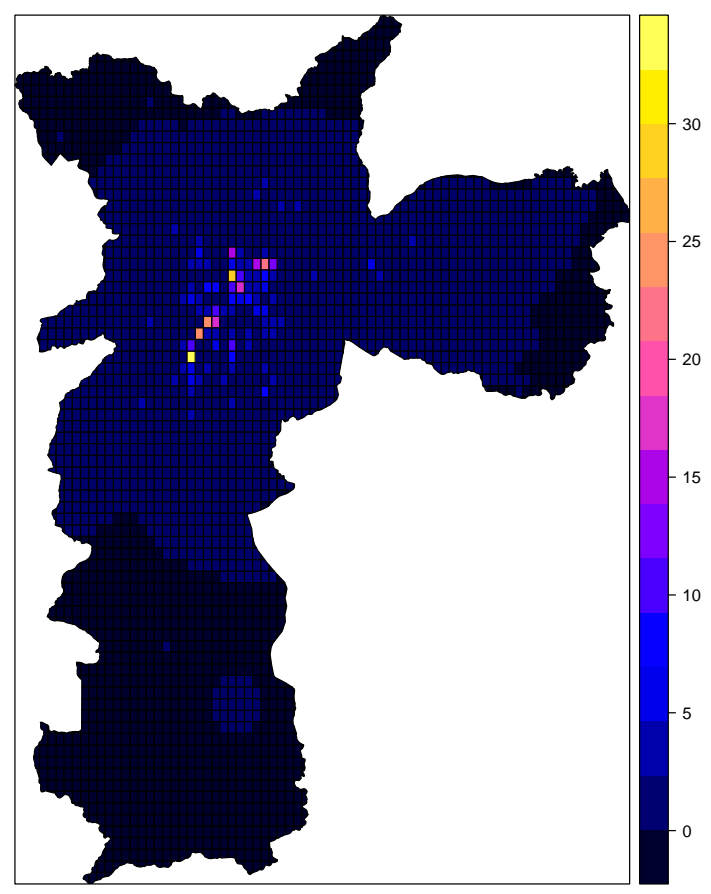

Figura 5.9 - Ajuste - LGCP irrestrito.

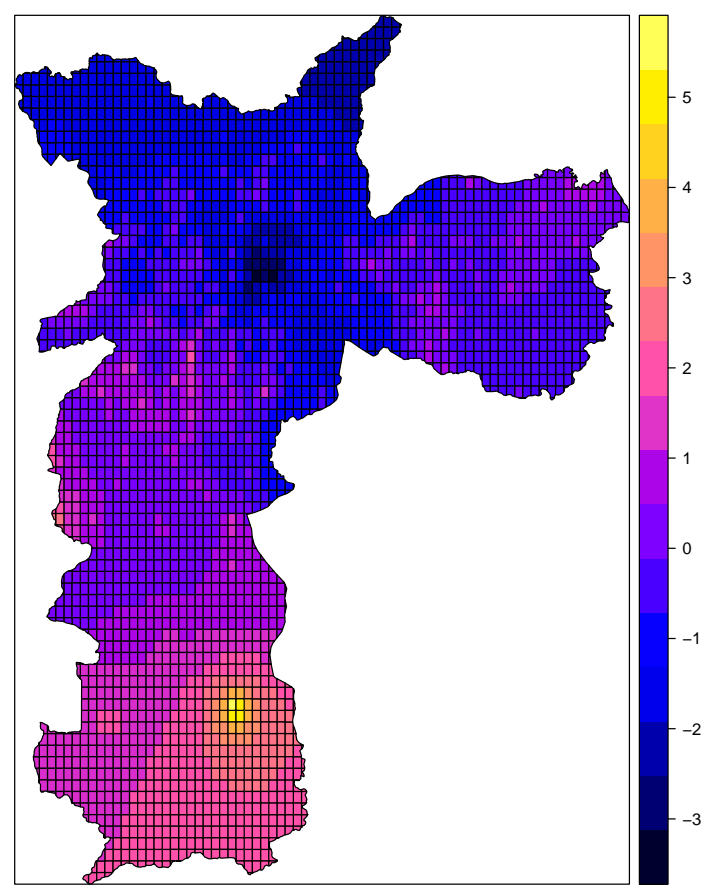

Figura 5.10 - Efeito estruturado capturado pelo modelo LGCP restrito. 


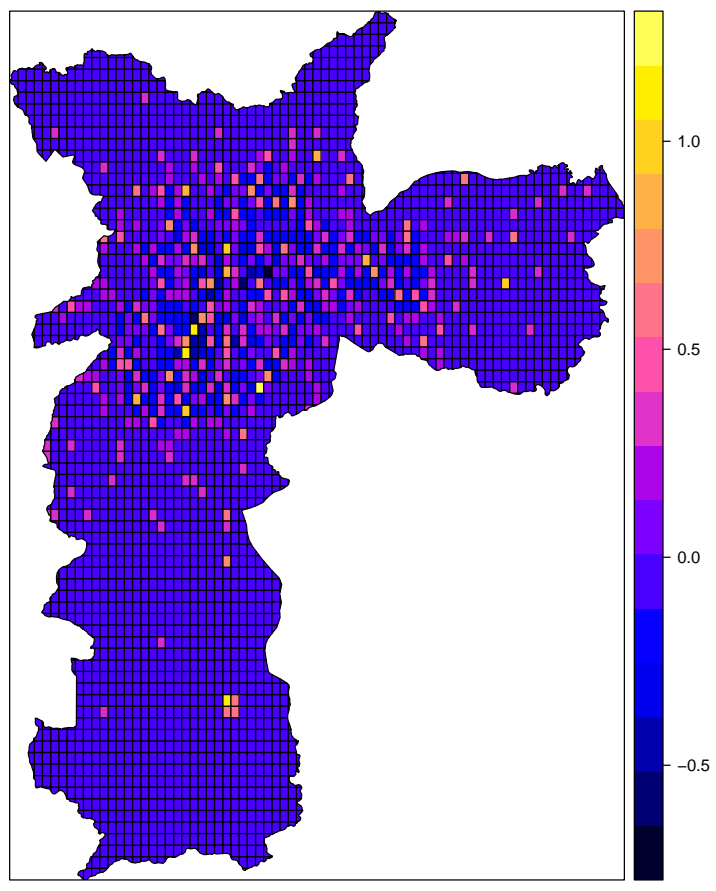

Figura 5.11 - Efeito não estruturado capturado pelo modelo LGCP restrito.

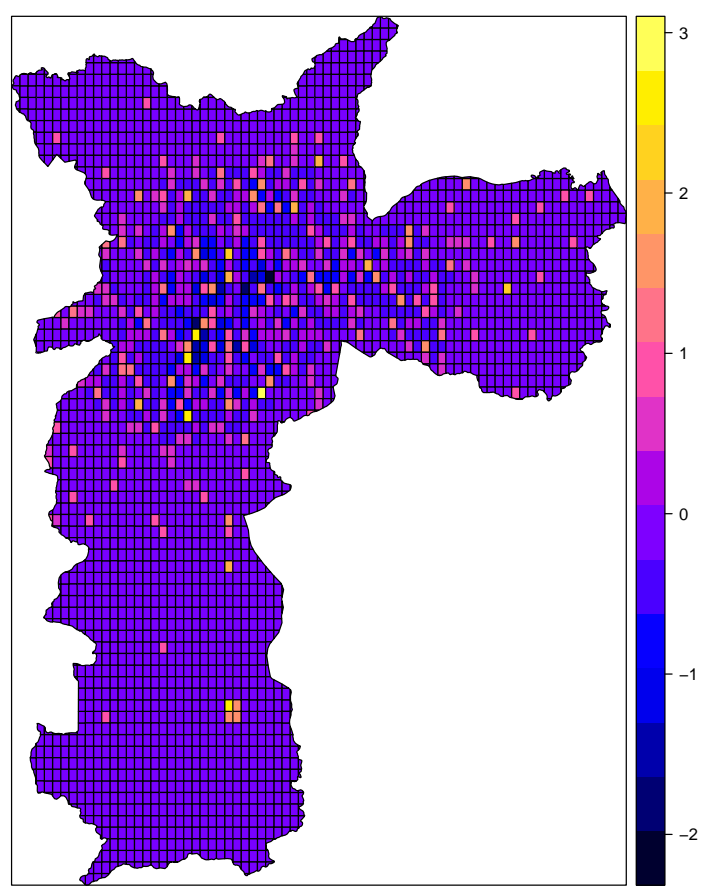

Figura 5.12 - Erro do modelo LGCP restrito: predito menos observado. 


\begin{tabular}{rrrrrr}
\hline & ME & RMSE & MAE & MPE & MAPE \\
\hline Poisson & 0.00091 & 1.34192 & 0.42012 & 13.59598 & 157.21390 \\
LGCP Irres & 0.02455 & 0.37369 & 0.19710 & 66.53538 & 103.46675 \\
LGCP Res & 0.02541 & 0.37488 & 0.19714 & 66.51052 & 103.51919 \\
\hline
\end{tabular}

Tabela 5.7 - Estatística de ajuste dentro da amostra

Podemos observar que o modelo de Poisson apresenta erros médios menores, mas é muito pior nos critérios baseados em erros quadráticos e absolutos, indicando o ganho no uso da especificação de intensidade estocástica com o controle de dependência espacial obtido com o uso dos modelos LGCP. Também é possível notar que não há perda significativa de ajuste com o uso do modelo restrito em relação ao modelo irrestrito nas especificações com base no processo LGCP.

Em geral, os resultados da estimativa indicam que a especificação do modelo LGCP é superior ao modelo de Poisson, devido à importância do efeito aleatório espacial incluído neste processo. Este componente pode ser interpretado como uma aproximação aos efeitos de atração e repulsão não capturados pelas covariadas que estão no modelo, já que o modelo de Poisson é condicionalmente independente. Este efeito espacial também pode ser interpretado como uma forma de agregar todas as demais variáveis omitidas, resumindo em um único fator os efeitos agregados dessas variáveis que levam a processos de dependência espacial. Por outro lado, pode ser que exista uma limitação no conjunto de variáveis que foram utilizadas nesta pesquisa dado que a maioria delas estão relacionadas a aspectos de infra-estrutura e desenvolvimento urbano. 


\section{Conclusão}

Esta pesquisa teve o objetivo de mostrar novos aplicações de estatística espacial no contexto de economia urbana, propondo o uso de novas metodologias de modelagem de processos pontuais espaciais e também como incorporar diversas fontes de dados neste problema. Os obtidos resultados mostraram-se encorajadores para extrair novas informações sobre processos de localização espacial e abrem a possibilidade de novas aplicações.

Estudos como esse são importantes para o avanço da literatura de economia urbana e também para a tomada de decisões em políticas públicas. Um aspecto fundamental em análise e desenho de políticas públicas está no uso eficiente das informações disponíveis. Nesse contexto nosso trabalho permite utilizar combinar informações espaciais de diversos formatos, como dados projetados continuamente no espaço (informações de satélite como luminosidade noturna e outras fontes), medidas contínuas de densidade, como densidade de linhas de ônibus, e informações baseadas em distâncias e dados de unidades areais, como a informação de renda per capita de distritos. Nas análises tradicionais de econometria espacial, os dados são normalmente agregados para unidades censitárias, como distritos ou bairros, desperdiçando a riqueza informacional presente em processos no contínuo espacial, como o processo de localização de empresas individuais analisado no presente trabalho.

Foram feitos nesta pesquisa três diferentes exercícios de estimação utilizando o método proposto de análise espacial, verificando o desempenho do modelo de Poisson não-homogêneo e especificações irrestritas e restritas do processo de Cox log gaussiano. Os resultados indicam a importância de medidas de infraestrutura e desenvolvimento urbano para o processo de atração de empresas, e especialmente mostram como o processo de aglomeração de indústrias ocorre pode ser modelado via um efeito espacial incluído na especificação de um processo de intensidade estocástico em um modelo de Cox espacial.

O bom desempenho dos modelos LGCP indicam que este processo pode ser aplicado com sucesso neste contexto de economia urbana, indicando sua possível utilizado em todos os contextos de processos de ocorrência/localização espacial relacionados a processos econômicos. Essa mesma metodologia pode ser aplicada para outros tipos de empresas apenas modificando o código CNAE de seleção de amostra, indicando o escopo possível de extensões desta mesma análise.

Outra aplicação importante para esta metodologia é o estudo dos determinantes econômicos da ocorrência espacial de crimes. As mesmas variáveis utilizadas neste trabalho podem ser utilizadas no estudo dos determinantes econômicos da criminalidade, como as variáveis relacionadas a infraestrutura e desenvolvimento urbano, e algumas tem uma interpretação ainda mais direta, como por exemplo a luminosidade noturna, que é um indicador importante de segurança urbana. Desta forma esperamos que este trabalho permita novas aplicações de processos pontuais espaciais e mecanismos de combinação de dados em outras aplicações em economia urbana e 
áreas relacionadas. 


\section{Referências}

ARAÚJO, M. d. F. Mapa da estrutura industrial e comercial do estado de são paulo. São Paulo em perspectiva, Fundação Seade São Paulo, v. 13, n. 1-2, p. 40-52, 1999.

ARAUZO-CAROD, J.-M.; LIVIANO-SOLIS, D.; MANJÓN-ANTOLÍN, M. Empirical studies in industrial location: an assessment of their methods and results. Journal of Regional Science, Wiley Online Library, v. 50, n. 3, p. 685-711, 2010.

BADDELEY, A.; BÁRÁNY, I.; SCHNEIDER, R. Spatial point processes and their applications. Lecture Notes in Mathematics, Springer-Verlag, v. 1892, 2007.

BADDELEY, A.; RUBAK, E.; TURNER, R. Spatial Point Patterns: Methodology and Applications with R. [S.l.]: CRC Press, 2015.

BARLET, M.; BRIANT, A.; CRUSSON, L. Location patterns of service industries in France: A distance-based approach. Regional Science and Urban Economics, Elsevier, v. 43, n. 2, p. 338-351, 2013.

BECKMANN, M. J.; THISSE, J.-F. The location of production activities. In: DURANTON, G.; HENDERSON, V. J.; STRANGE, W. C. (Ed.). Handbook of Regional and Urban Economics. [S.1.]: Elsevier, 1987. v. 1, p. 21-95.

BEHRENS, K.; MION, G.; MURATA, Y.; SÜDEKUM, J. Spatial frictions. Journal of Urban Economics, Elsevier, v. 97, p. 40-70, 2017.

BESAG, J. Spatial interaction and the statistical analysis of lattice systems. Journal of the Royal Statistical Society. Series B (Methodological), v. 36, n. 2, p. 192-236, 1974.

BESAG, J. Statistical analysis of non-lattice data. The Statistician, v. 24, n. 3, p. 179-195, 1975.

BESAG, J. Some methods of statistical analysis for spatial data. Bulletin of the International Statistical Institute, v. 47, n. 2, p. 77-92, 1977.

BIVAND, R. S.; PEBESMA, E. J.; GOMEZ-RUBIO, V.; PEBESMA, E. J. Applied Spatial Data Analysis with R. [S.1.]: Springer, 2008.

BLANGIARDO, M.; CAMELETTI, M. Spatial and Spatio-Temporal Models with R-INLA. [S.1.]: Wiley, 2015.

BOCCI, C.; ROCCO, E. Modelling the location decisions of manufacturing firms with a spatial point process approach. Journal of Applied Statistics, Taylor \& Francis, v. 43, n. 7, p. 1226-1239, 2016.

BRUECKNER, J. K. The structure of urban equilibria: A unified treatment of the muth-mills model. In: DURANTON, G.; HENDERSON, V. J.; STRANGE, W. C. (Ed.). Handbook of Regional and Urban Economics. [S.1.]: Elsevier, 1987. v. 2, p. 821-845.

CARVALHO, M. S.; SOUZA-SANTOS, R. Análise de dados espaciais em saúde pública: métodos, problemas, perspectivas analysis of spatial data in public health: methods, problems, and perspectives. Cad. Saúde Pública, SciELO Brasil, v. 21, n. 2, p. 361-378, 2005. 
CHAPMAN, L.; THORNES, J. E. The use of geographical information systems in climatology and meteorology. Progress in physical geography, Sage Publications Sage CA: Thousand Oaks, CA, v. 27, n. 3, p. 313-330, 2003.

CHEN, X.; NORDHAUS, W. D. Using luminosity data as a proxy for economic statistics. Proceedings of the National Academy of Sciences, National Acad Sciences, v. 108, n. 21, p. 8589-8594, 2011.

CHIU, S. N.; STOYAN, D.; KENDALL, W. S.; MECKE, J. Stochastic Geometry and it's Applications. [S.1.]: John Wiley \& Sons Ltd, 2013.

CICCONE, A.; HALL, R. E. Productivity and the density of economic activity. [S.1.], 1993.

CRESSIE, N. A. Statistics for Spatial Data. [S.1.: s.n.], 1993.

DESMET, K.; ROSSI-HANSBERG, E. Urban accounting and welfare. American Economic Review, v. 103, n. 6, p. 2296-2327, 2013.

DESMET, K.; ROSSI-HANSBERG, E. Analyzing urban systems: have megacities become too large? Unpublished working paper. 2014.

DIGGLE, P. A kernel method for smoothing point process data. Applied Statistics, JSTOR, p. 138-147, 1985.

DIGGLE, P. J. Statistical Analysis of Spatial Point Processes. [S.l.: s.n.], 1983.

DOLL, C. N.; MULLER, J.-P.; MORLEY, J. G. Mapping regional economic activity from night-time light satellite imagery. Ecological Economics, Elsevier, v. 57, n. 1, p. 75-92, 2006.

DURANTON, G.; PUGA, D. Micro-foundations of urban agglomeration economies. In: DURANTON, G.; HENDERSON, V. J.; STRANGE, W. C. (Ed.). Handbook of Regional and Urban Economics. [S.1.]: Elsevier, 2004. v. 4, p. 2063-2117.

ELLISON, G.; GLAESER, E. L.; KERR, W. R. What causes industry agglomeration? evidence from coagglomeration patterns. American Economic Review, v. 100, n. 3, p. 1195-1213, 2010.

ELVIDGE, C. D.; SUTTON, P. C.; GHOSH, T.; TUTTLE, B. T.; BAUGH, K. E.; BHADURI, B.; BRIGHT, E. A global poverty map derived from satellite data. Computers \& Geosciences, Elsevier, v. 35, n. 8, p. 1652-1660, 2009.

FERNANDES, A. C.; CÔRTES, M. R.; PINHO, M. Caracterização das pequenas e médias empresas de base tecnológica em são paulo: uma análise preliminar. Economia e Sociedade, v. 13, n. 1, p. 151-173, 2004.

GLAESER, E. L. Cities, agglomeration, and spatial equilibrium. [S.1.]: Oxford University Press, 2008.

GÓMEZ-RUBIO, V.; PALMÍ-PERALES, F. Spatial models with the Integrated Nested Laplace Approximation within Markov Chain Monte Carlo. arXiv preprint arXiv:1702.03891, 2017.

GOODCHILD, M. F.; ANSELIN, L.; DEICHMANN, U. A framework for the areal interpolation of socioeconomic data. Environment and Planning A, SAGE Publications Sage UK: London, England, v. 25, n. 3, p. 383-397, 1993. 
GORDON, I. R.; MCCANN, P. Industrial clusters: complexes, agglomeration and/or social networks? Urban Studies, Sage Publications Sage UK: London, England, v. 37, n. 3, p. 513-532, 2000 .

GREENSTONE, M.; HORNBECK, R.; MORETTI, E. Identifying agglomeration spillovers: Evidence from winners and losers of large plant openings. Journal of Political Economy, The University of Chicago Press, v. 118, n. 3, p. 536-598, 2010.

HANSEN, E. R. Industrial location choice in sao paulo, brazil: a nested logit model. Regional science and Urban economics, Elsevier, v. 17, n. 1, p. 89-108, 1987.

HELSLEY, R. W.; STRANGE, W. C. Coagglomeration, clusters, and the scale and composition of cities. Journal of Political Economy, University of Chicago Press Chicago, IL, v. 122, n. 5, p. 1064-1093, 2014.

HENDERSON, J. V.; STOREYGARD, A.; WEIL, D. N. Measuring economic growth from outer space. The American Economic Review, American Economic Association, v. 102, n. 2, p. 994-1028, 2012.

HOOVER, E. M. Location Theory and the Shoe Leather Industries. [S.1.]: Harvard University Press, 1937.

HOOVER, E. M. The Location of Economic Activity. [S.l.]: Mcgraw-Hill Book Company, Inc; London., 1948.

ILLIAN, J. B.; SØRBYE, S. H.; RUE, H. A toolbox for fitting complex spatial point process models using Integrated Nested Laplace Approximation (inla). The Annals of Applied Statistics, JSTOR, p. 1499-1530, 2012.

KOLKO, J. Urbanization, agglomeration, and coagglomeration of service industries. In: Agglomeration economics. [S.1.]: University of Chicago Press, 2010. p. 151-180.

LAURINI, M. P. Income estimation using night luminosity: A continuous spatial model. Spatial Demography, v. 4, n. 2, p. 83-115, 2016.

LINDGREN, F.; RUE, H. Bayesian spatial modelling with r-inla. Journal of Statistical Software, University of Bath, v. 63, n. 19, 2015.

LUCAS, R. E.; ROSSI-HANSBERG, E. On the internal structure of cities. Econometrica, Wiley Online Library, v. 70, n. 4, p. 1445-1476, 2002.

MARSHALL, A. Principles of political economy. Maxmillan, New York, 1890.

MESSNER, S. F.; ANSELIN, L. Spatial analyses of homicide with areal data. Spatially Integrated Social Science, v. 12, 2004.

MILLS, E. S. An aggregative model of resource allocation in a metropolitan area. The American Economic Review, JSTOR, v. 57, n. 2, p. 197-210, 1967.

MØLLER, J.; SYVERSVEEN, A. R.; WAAGEPETERSEN, R. P. Log gaussian Cox processes. Scandinavian Journal of Statistics, Wiley Online Library, v. 25, n. 3, p. 451-482, 1998.

MOOMAW, R. L. Productivity and city size: a critique of the evidence. The Quarterly Journal of Economics, MIT Press, v. 96, n. 4, p. 675-688, 1981. 
MORETTI, E. Workers' education, spillovers, and productivity: evidence from plant-level production functions. American Economic Review, v. 94, n. 3, p. 656-690, 2004.

MOSES, L. N. Location and the theory of production. The Quarterly Journal of Economics, JSTOR, v. 72, n. 2, p. 259-272, 1958.

OHLIN, B. International and Interregional Trade. [S.1.: s.n.], 1933.

ORCUTT, G. H.; WATTS, H. W.; EDWARDS, J. B. Data aggregation and information loss. The American Economic Review, JSTOR, p. 773-787, 1968.

O'SULLIVAN, D.; MORRISON, A.; SHEARER, J. Using desktop gis for the investigation of accessibility by public transport: an isochrone approach. International Journal of Geographical Information Science, Taylor \& Francis, v. 14, n. 1, p. 85-104, 2000.

RESENDE, M. Industrial coagglomeration: some state-level evidence for brazil. Nova Economia, SciELO Brasil, v. 25, n. 1, p. 181-194, 2015.

RIBEIRO, M. A. C.; ALMEIDA, R. d. Padrões de localização espacial e estrutura de fluxos dos estabelecimentos industriais da área metropolitana de recife. Revista Brasileira de Geografia, v. 42 , n. 2, p. 203-264, 1980.

RIETVELD, P. Spatial economic impacts of transport infrastructure supply. Transportation Research Part A: Policy and Practice, Elsevier, v. 28, n. 4, p. 329-341, 1994.

ROSENTHAL, S. S.; STRANGE, W. C. Evidence on the nature and sources of agglomeration economies. In: DURANTON, G.; HENDERSON, V. J.; STRANGE, W. C. (Ed.). Handbook of Regional and Urban Economics. [S.1.]: Elsevier, 2004. v. 4, p. 2119-2171.

RUE, H.; MARTINO, S.; CHOPIN, N. Approximate bayesian inference for latent gaussian models by using integrated nested Laplace approximations. Journal of the Royal Statistical Society: Series B (Statistical Methodology), Wiley Online Library, v. 71, n. 2, p. 319-392, 2009.

WEBER, A. Theory of industrial location. [S.1.]: University of Chicago Press, Chicago, 1909.

WHEAT, L. F. The effect of modern highways on urban manufacturing growth. Highway Research Record, n. 277, 1969. 